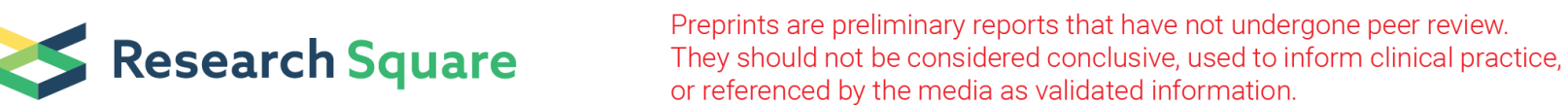

\section{Transcriptomic analysis at organ and time scale reveals gene regulatory networks controlling the sulfate starvation response of Solanum lycopersicum.}

Javier Canales ( $\nabla$ javier.canales@uach.cl)

Universidad Austral de Chile - Campus Isla Teja https://orcid.org/0000-0002-6263-6974

Felipe Uribe

Universidad Austral de Chile - Campus Isla Teja

Carlos Henríquez-Valencia

Universidad Austral de Chile - Campus Isla Teja

Carlos Lovazzano

Universidad Austral de Chile - Campus Isla Teja

Joaquín Medina

Centro de Biotecnologia y Genomica de Plantas

Elena A. Vidal

Universidad Mayor

Research article

Keywords: Sulfate, transcriptomics, gene networks, transcription factors, starvation, roots, leaves, tomato, Solanum lycopersicum, SLIM1

Posted Date: August 11th, 2020

DOI: https://doi.org/10.21203/rs.3.rs-31412/v2

License: (c) (i) This work is licensed under a Creative Commons Attribution 4.0 International License.

Read Full License

Version of Record: A version of this preprint was published at BMC Plant Biology on August 24th, 2020. See the published version at https://doi.org/10.1186/s12870-020-02590-2. 


\section{Abstract}

Background: Sulfur is a major component of biological molecules and thus an essential element for plants. Deficiency of sulfate, the main source of sulfur in soils, negatively influences plant growth and crop yield. The effect of sulfate deficiency on plants has been well characterized at the physiological, transcriptomic and metabolomic levels in Arabidopsis thaliana and a limited number of crop plants. However, we still lack a thorough understanding of the molecular mechanisms and regulatory networks underlying sulfate deficiency in most plants. In this work we analyzed the impact of sulfate starvation on the transcriptome of tomato plants to identify regulatory networks and key transcriptional regulators at a temporal and organ scale.

Results: Sulfate starvation reduces the growth of roots and leaves which is accompanied by major changes in the organ transcriptome, with the response being temporally earlier in roots than leaves. Comparative analysis showed that a major part of the Arabidopsis and tomato transcriptomic response to sulfate starvation is conserved between these plants and allowed for the identification of processes specifically regulated in tomato at the transcript level, including the control of internal phosphate levels. Integrative gene network analysis uncovered key transcription factors controlling the temporal expression of genes involved in sulfate assimilation, as well as cell cycle, cell division and photosynthesis during sulfate starvation in tomato roots and leaves. Interestingly, one of these transcription factors presents a high identity with SULFUR LIMITATION1, a central component of the sulfate starvation response in Arabidopsis.

Conclusions: Together, our results provide the first comprehensive catalog of sulfate-responsive genes in tomato, as well as novel regulatory targets for future functional analyses in tomato and other crops.

\section{Background}

Sulfur (S) is an essential nutrient for living organisms, as main component of important proteinogenic amino acids methionine (Met) and cysteine (Cys), and of numerous coenzymes and prostethic groups such as iron-sulfur centers, S-adenosylmethionine (SAM), glutathione and coenzyme-A, among others [1, 2]. As such, $S$ is directly involved in a wide variety of metabolic processes in plants, including photosynthesis or nitrate reduction and assimilation [2]. $\mathrm{S}$ is also a component of the thioglucosides glucosinolates (GSLs) in Brassicales and allyl Cys sulfoxides in Allium species, important secondary metabolites involved in defense responses [1,3]. Importantly, plants are a main source of reduced $S$ for animals and humans, who are unable to assimilate inorganic $S$ and must obtain it from organic compounds such as proteins in their diet. As a macronutrient, the average concentration of $S$ in plant tissues for adequate growth is relatively high (around 0.1 to $0.5 \%$ of plant dry weight) [4]. However, $S$ deficiency has become a rising problem in crops primarily due to decreases in atmospheric $S$ inputs caused by strict industrial emission regulations and changes in agronomic practices, including the use of fertilizers with reduced S compositions, reduction in the use of S-containing fungicides and intensive cropping systems that remove $S$ from the soils [5]. Prolonged $S$ deficiency causes a reduction in 
photosynthetic rate, chlorophyll levels and amino acid contents, leading to growth retardation and diminished yield, nutritional quality and taste of crops [5-7]. Moreover, S-starvation alters the acquisition of other nutrients such as nitrate, phosphate, molybdenum, selenium, and iron [8-11]. In this framework, understanding the regulation of the S-starvation response and how it impacts the acquisition and metabolism of S, as well as other processes in plants has become a major interest for research and crop improvement.

Sulfate is the most stable form of $S$ in soils, and thus the main source of $S$ for plants [1]. Sulfate is taken up by root epidermal cells via the action of high affinity sulfate transporters (SULTRs) [12], mainly SULTR1;1 and SULTR1;2 in Arabidopsis [13,14]. Sulfate can then be transported to the aerial tissues by specific transporters such as SULTR2;1 and SULTR3;5 or stored in vacuoles through the action of SULTR4;1 and SULTR4;2 [15]. The first step in sulfate assimilation is catalyzed by ATP sulfurylase (ATPS), generating adenosine 5'-phosphosulfate (APS). At this point, APS can be reduced by APS reductase (APR) and sulfite reductase to sulfide, which is incorporated into 0 -acetylserine (OAS) to form cysteine, or can be phosphorylated by APS kinase to 3'-phosphoadenosine 5'-phosphosulfate (PAPS) [1, 2]. PAPS, can be utilized as a sulfate donor to synthesize a variety of sulfated metabolites such as GSLs, brassinosteroids, sulfoflavonoids, phytosulfokines and sulfojasmonates [3].

Sulfate deficiency or starvation triggers important changes in sulfate acquisition and metabolism in plants, such as the catabolism of S-storage compounds and repression of processes involved in the biosynthesis of secondary S metabolites [16]. These changes involve upregulation of sulfate transporters, accumulation of OAS and degradation of GSLs [16]. This complex rearrangement of plant metabolism is partly explained by changes that occur at the transcriptional and post-transcriptional levels. At the transcriptional level, S-responsive elements (SUREs) sequences have been identified in the promoter regions of SULTR genes, APR genes and of other sulfate deficiency-controlled genes [17-20]. In the last years, multiple efforts have been carried out to identify the regulatory factors that control the expression of sulfate starvation-responsive genes. Using genetic approaches, different transcription factors (TFs) have been shown to be involved in sulfate transport and/or assimilation control, including MYB family members, LONG HYPOCOTYL 5 (HY5), and the EIL family TF SULFUR LIMITATION 1 (SLIM1) [21-24]. SLIM1 has arisen as an important TF controlling the sulfate deficiency response [25]. This TF is a transcriptional regulator of sulfate transporters and GSL synthesis genes, among other sulfate-deficiency controlled genes in Arabidopsis [24, 25]. At the post-transcriptional level, microRNA395 (miR395) targets the low-affinity transporter SULTR2;1 and three members of the ATPS gene family [26]. miR395 is highly induced by sulfate starvation and acts downstream SLIM1 to control sulfate assimilation and homeostasis [27-30]. Moreover, the nuclear protein MORE SULPHUR ACCUMULATION 1 (MSA1) has been shown to control SAM biosynthesis, affecting global DNA methylation and causing the hypomethylation of the SULTR1;1 and SULTR1;2 transporter genes and the APR3 gene, indicating a possible epigenetic mechanism of control of $S$ homeostasis in the plant [31].

The utilization of -omics approaches to study plant responses to $S$ availability in the last decades has significantly advanced our understanding of the molecular mechanisms and physiological processes 
regulating S metabolism and homeostasis in plants [1,32]. A myriad of genes differentially expressed in response to sulfate deficiency have been identified using transcriptomic approaches, mostly in Arabidopsis [10, 17, 18, 24, 33-39] and Triticum aestivum [40-43]. Meta-analysis of transcriptomics data

has shown that sulfate deficiency-responsive genes are enriched in biological processes related to sulfate transport and metabolism, but also in processes related to cell wall organization, regulation of proteolysis, and metabolism of carbon and nitrogen [44]. The sulfate deficiency response has also been explored by metabolomics and proteomics analyses in Arabidopsis and crops [10, 18, 33, 36, 45-53]. Furthermore, systems biology approaches that integrate multi-omics data have been adopted to generate a holistic vision of the sulfate-deficiency response [32]. These integrative approaches have allowed for the identification of TF candidates involved in the sulfate-deficiency response such as MYB28 and MYB29 [45, 54], IAA28 [55, 56], NF-YA2 and RVE2 [44], among others [18].

Tomato (Solanum lycopersicum) is one of the most important vegetable crops in the world [57]. In 2018, the total world area harvested for tomatoes was 4.7 million ha, with an annual production quantity of 182 million tons (http://fao.org/faostat/en/\#data/QC). Tomato produces fleshy fruits important for the human diet as a source of vitamins and carotenes such as beta-carotene and lycopene [58]. Sulfate deficiency severely reduces the growth of tomato plants [59-63], diminishing biomass, protein concentration, total $S$ in shoots and roots [62, 63], chlorophyll contents [60-62], as well as yield [64]. Given the economic importance of tomato worldwide, understanding how tomato responds to sulfate starvation at the molecular level and identifying key regulatory components is of paramount importance for sustainable tomato production in the present and future agricultural scenarios.

In this work, we identified genes and biological processes that participate in the sulfate starvation response of tomato, at a temporal and organ scale using transcriptomics and network analysis. Integration of expression and regulatory TF-target interaction data allowed us to pinpoint key candidate TFs that might coordinate the temporal growth response of roots and leaves to external sulfate availability.

\section{Results}

Sulfate starvation has a major impact on leaf and root growth in early stages of tomato development

As previously reported for other tomato cultivars [59-64], we found that the growth of Solanum lycopersicum cv. Moneymaker seedlings was severely impacted by sulfate starvation. As shown in Fig. $1 \mathrm{~A}$, lack of external sulfate has a clear negative effect on the growth of the aerial tissue, as compared with the full nutrient condition. This effect is noticeable from the third week after sowing. Multifactorial analysis of leaf fresh weight and total leaf area showed that there is a strong interaction between sulfate availability and time factors ( $\mathrm{p}$-value $<0.001$, two-way ANOVA, Fig. $1 \mathrm{~B}$ and Figure $\mathrm{S} 1$ ), indicating that the growth response of leaves to sulfate availability depends on the plant developmental stage. Accordingly, no significant differences between control and sulfate-starved plants were observed at 2 weeks after sowing ( $p$-value $=0.81$ ), while a strong dependence of external sulfate on leaf fresh weight and area was 
observed 3 and 4 weeks after sowing (p-value <0.001) (Fig. 1B and Figure S1). The same interaction between sulfate and time was observed for root weight, with differences between control and sulfatestarved plants being significant only after 3 and 4 weeks of sowing ( $p$-value $<0.001$, Fig. $1 \mathrm{C}$ ). These results indicate that whole-plant growth is severely affected by the lack of external sulfate from 3 weeks after sowing.

Consistent with leaf weight and leaf area measurements over time, there is a steady accumulation of sulfate in leaves, that is abolished in sulfate-starved plants in weeks 3 and 4 (Fig. 1D). In contrast, the increase in root weight over time of plants grown in control condition is not explained by a concurrent increase in root sulfate levels (Fig. 1E). Moreover, no major differences in sulfate accumulation are observed in the roots of full nutrient or sulfate-starved plants (Fig. 1E). Together, these results indicate that additional factors besides sulfate content might be controlling root growth response to sulfate starvation.

Sulfate starvation promotes major changes in leaf and root transcriptomes in a time-dependent manner

In order to gain insights into the molecular mechanisms underlying leaf and root growth repression in response to sulfate starvation, we performed a transcriptomic analysis at a temporal and organ scale, using RNA-seq. To perform this analysis, total RNA from roots and leaves was extracted at 2, 3 and 4 weeks after sowing, considering three independent biological replicates. The RNA-Seq data was pseudoaligned to the ITAG3.20 transcriptome annotation using kallisto [65], and normalized expression data in transcripts per million (TPM) was obtained (Table S1).

Principal component analysis (PCA) of the RNA-seq data showed that samples are well separated along the first component, which explains more than $50 \%$ of the variability in leaf and root samples (Fig. 2). Interestingly, full nutrient and sulfate-starved samples from leaves are grouped together at 2 weeks after sowing, indicating similar transcript expression profiles at this time point (Fig. 2). In contrast, control and sulfate-starved samples from root tissue at 2 weeks are well separated, suggesting that sulfate starvation alters the plant transcriptome in an organ- and time-dependent fashion, with an earlier response in root tissue.

Accordingly, no genes were found differentially expressed between control and sulfate-starved leaves at two weeks after sowing, while 438 genes were differentially expressed in roots in this time point $\left(\log _{2}\right.$ Fold Change $>1$ and q-value $<0.05$ ) (Figure S2A and Table S2). In addition, 55\% of these 438 genes are subsequently regulated in leaves ( 241 genes, Figure S2B), which point out an orchestrated whole organism response to sulfate deprivation over developmental time.

A considerable number of genes were differentially expressed by sulfate starvation in roots and leaves: 7024 genes at 3 weeks after sowing and 6163 at 4 weeks after sowing, with most genes being differentially expressed in leaves (Figure S2A and Table S2). This major reprogramming of the plant transcriptome correlates with the observed differences in leaf and root growth of control and sulfate- 
deprived plants between weeks 3 and 4, suggesting changes in gene expression are partly responsible for the observed phenotypes (Fig. 1A and B).

In order to identify genes that are differentially expressed in response to sulfate starvation, time or a sulfate starvation-time interaction, we performed a multifactorial analysis of RNA-seq data in each tissue using sleuth [66]. In the case of roots, 6052 genes were significantly affected by time, 3755 genes by sulfate starvation and 714 by the interaction of both factors (q-value $<0.05$, and $\log _{2} F C>1$ ) (Fig. $3 \mathrm{~A}$ ). In the case of leaves, 6084 genes were affected by sulfate starvation, 7062 genes by time and 4571 genes by the interaction of both factors (q-value $<0.05$, and $\log _{2} F C>1$ ) (Fig. 3B). In contrast to root tissue, these results indicate that the response to sulfate starvation in leaves has a strong dependence on the plant age. In fact, about $90 \%$ of sulfate-responsive genes in leaves are significantly modulated by time (Fig. 3B).

To validate the results of the RNA-seq analysis with an alternative RNA quantification methodology, we selected 10 sulfate-regulated genes in root and leaves involved in different molecular and biological functions including sulfate metabolism, transport and transcriptional regulation, as well as a group of genes with unknown functions (Table S3). The expression patterns obtained by qPCR analysis showed a significant positive correlation with RNA-seq data ( $P<0.0001 ; 0.89$ Pearson correlation value; Figure S3), indicating that most of genes analyzed showed a similar expression pattern than the ones identified by RNA-seq analysis.

Comparative analysis of the tomato and Arabidopsis sulfate starvation-responsive transcriptome

In a previous study, we identified 2046 genes regulated by sulfate availability in Arabidopsis thaliana using an integrative meta-analysis of transcriptomic data [44]. This meta-analysis included samples of roots and leaves at different stages of development (from seedling to juvenile plants). In the case of tomato plants, multifactorial analysis identified 7589 sulfate-responsive genes in roots and leaves. In order to compare the sulfate starvation responsive transcriptome of both Arabidopsis and tomato, we first performed an orthology analysis of sulfate responsive genes. The identification of orthologous groups allows cross-referencing of genes from multiple species [67]. We used the PLAZA 4.0 database [68] to identify the ortholog group to which each sulfate-regulated gene belongs. Thus, we identified 4262 orthologous gene families out the 7589 sulfate-responsive genes in tomato and 1338 orthologous gene families out the 2046 sulfate-responsive genes in Arabidopsis (Table S4). We found that $70 \%$ of the Arabidopsis orthologous gene families that are associated with sulfate-responsive genes were also present in tomato plants (Fig. 4A). This result indicates that the RNA-seq analysis in tomato plants captured most of the sulfate-responsive gene families previously detected in Arabidopsis.

Over-representation analysis of biological functions shared by both species revealed several GO terms that are expected to be conserved in the sulfate response as "cellular oxidant detoxification", "sulfate transmembrane transport" or "sulfur utilization" (Table S5). Interestingly, we also found a significant enrichment (adjusted p-value $<0.05$ ) in $\mathrm{GO}$ terms that have not been functionally analyzed in previous 
studies in the context of the sulfate starvation response such as those related to cell wall or cytokinin transport (Table S5). On the other hand, specific GO terms of the Brassicaceae family such as GSL biosynthesis are among the most over-represented biological processes in the case of sulfate-responsive genes in Arabidopsis (Fig. 4B and Table S5). The intersection between the lists of orthologue genes of both species also detected sulfate-responsive processes that are found exclusively in tomato plants. The most over-represented biological process in this group of genes was "cellular response to phosphate starvation" (Fig. 4C and Table S5), suggesting that sulfate deficiency also affects internal phosphate levels in tomato plants. Specifically, we found 52 genes in this biological process including SPX genes [69], phosphatases and genes encoding enzymes involved in phosphate mobilization by membrane lipid remodeling such as UDP-sulfoquinovose synthase [70, 71] (Figure S4). In order to determine the response of this set of phosphate-related genes to sulfate starvation in tomato plants, we computed the average fold of change between sulfate starved and control samples in both tissues and we then performed a hierarchical clustering analysis. We found that $60 \%$ of the genes involved in the phosphate starvation response (30 out 52 genes) showed lower expression levels in sulfate-starved plants at 3 and 4 weeks after sowing in both tissues (Cluster 3, Figure S4), suggesting that sulfate starvation alters internal phosphate levels. To address this hypothesis, we determined the internal phosphate levels at 3 weeks after sowing in both tissues. The results shown in Fig. 4D, indicate that sulfate-starved plants exhibit significantly higher phosphate levels than control plants in both tissues, with a higher phosphate accumulation in leaves.

\section{Temporal dynamics of gene expression of tomato roots and leaves are altered by sulfate starvation}

In order to get further insights about the temporal patterns of expression of the genes and associated biological processes affected by sulfate starvation, we computed Pearson correlation indexes for each pair of sulfate and time-regulated genes in roots and leaves. We performed a hierarchical clustering analysis using Dynamic TreeCut [72], and identified six co-expression clusters for genes regulated by sulfate and time in roots and leaves (Fig. 5A, Fig. 5B, Figure S5, Figure S6). We found that the majority of genes (60\% in roots and 72\% in leaves) are contained in Clusters 1 and 2 (Fig. 5A and Fig. 5B). We thus decided to analyze these clusters in more detail.

As shown in Fig. 5C and Fig. 5D, the expression profiles of Clusters 1 and 2 in roots and leaves show similar trends. Genes in Cluster 1 are repressed over time, with an earlier decrease in mRNA levels under sulfate starvation conditions, between weeks 2 and 3 . On the other hand, the expression of genes in Cluster 2 slightly increases between weeks 3 and 4 in control conditions, while an important increase in gene expression is shown between weeks 2 and 3 under sulfate starvation conditions (Fig. $5 \mathrm{C}$ and Fig. 5D). Although the expression patterns of the genes contained in Clusters 1 and 2 are similar between roots and leaves, the identity of these sulfate-responsive genes differs between these organs (Table S6 and Table S7). As such, we found different enriched biological processes associated with Clusters 1 and 2 in roots and leaves. In roots, Cluster 1 is enriched in genes associated to growth, such as "epidermal cell division" and "plant-type secondary cell wall biogenesis", as well as processes related to redox activity, such as "hydrogen peroxide catabolic process" and "cellular oxidant detoxification" (Fig. 5C and Table 
S8). In leaves, we found an enrichment of genes involved in photosynthesis, microtubule-based movement and several GO terms related with cell division such as "mitotic cell cycle checkpoint" or "mitotic spindle organization" (Table S9). GO terms related to photosynthesis are especially abundant in this cluster, so we analyzed in more detail the distribution of these genes across different categories of primary metabolism using Mapman4 annotation framework [73]. In the case of photosynthesis, most genes are involved in light reactions, followed by the Calvin-Benson cycle and sucrose/starch metabolism (Figure S7A). Cluster 1 genes are also involved in other aspects of primary metabolism such as nitrogen assimilation and amino acid biosynthesis as well as fatty acid biosynthesis (Figure S7A). A similar result was obtained when all differentially expressed genes by sulfate starvation in leaves were analyzed using Mapman4 (Figure S7B). On the other hand, genes related to photosynthesis were under-represented in roots (Figure S7C), while other metabolic processes such as cell wall, lipid or amino acid metabolism showed a similar representation in roots and leaves (Figure S7B and Figure 7C). In the case of Cluster 2, and consistent with the marked induction in gene expression triggered by sulfate starvation shown in this cluster, we found an overrepresentation of genes involved in biological processes related to the cellular response to S starvation such as APR genes (Solyc02g032860, Solyc02g080640 and Solyc03g031620), RESPONSE TO LOW SULFUR (LSU) genes (Solyc03g096760, Solyc03g096770, Solyc03g096780) or sulfate transporters (Solyc05g054740, Solyc04g054730, Solyc04g072760, Solyc12g056930) (Fig. 5C and Table S8). Cluster 2 in leaves is enriched in GO terms related with the response to stress and protein phosphorylation (Fig. 5D and Table S9). Interestingly, we did not find an overrepresentation of Sstarvation-related processes in Cluster 2 in leaves, as observed in roots. In fact, classical genes involved in the sulfate starvation response such as SULTR, ATPS, APR or LSU belong to Cluster 6 (Figure S6), suggesting an organ-specific expression of these genes in response to external $S$ during development.

In summary, sulfate starvation promotes significant changes in the temporal expression patterns of a large number of genes in roots and leaves, of which many are associated with functions related to cellular growth, photosynthesis and sulfate uptake and metabolism. Moreover, the comparative analysis of major gene co-expression clusters between roots and leaves indicates an organ-specific response of the tomato plants to sulfate starvation.

\section{Identification of transcription factors involved in the sulfate starvation response in tomato}

Our results point at major transcriptomic changes occurring in tomato roots and leaves in response to sulfate starvation. As described in Arabidopsis, some of these changes may be attributed to transcriptional regulation by TFs. Although some TFs controlling sulfate responses have been identified in Arabidopsis [32], to date there is no information about sulfate-responsive TFs and their role in responses to sulfate starvation in tomato. In order to identify key TFs controlling the response to sulfate starvation of tomato, we constructed gene regulatory networks for roots and leaves using co-expression data and regulatory information on TF-DNA interaction obtained from PlantRegMap [74] .

In the case of roots, we obtained a gene regulatory network consisting of 24 TFs and 172 targets (Figure S8 and Table S10). TFs of the root regulatory network belong to 17 different families (Table S11), with 
MYB and bZIP TFs being the most abundant families (4 and 3 members, respectively). In the same way, we constructed a TF-target network using the identified sulfate-regulated genes in leaves which was composed by 45 TFs and 566 targets (Figure S9 and Table S12). Specifically, ERF, TCP and WRKY were the most abundant TF families in the leaves network with 7 and 5 members, respectively (Table S11). Notably, the most abundant TF families in the regulatory network of leaves were not shared with the root network (Table S11), suggesting organ-specific regulation of the transcriptional response to sulfate starvation.

We next focused our analysis on TFs whose predicted target genes have overrepresented biological functions (adjusted p-value $<0.05$ ), in order to correlate TF regulation to changes in functional processes. As shown in Fig. 6A and Fig. 6B, we found 3 TFs in roots and 10 TFs in leaves that potentially control genes involved in the sulfate starvation response, phytohormone biosynthesis and signaling, senescence, among other biological processes. Most connected factors in terms of outdegree were a MYB TF (Solyc11g071300) in roots and TCP 21 TF (Solyc03g006800) in leaves, showing 40 and 71 targets respectively (Fig. 6A and Fig. 6B). Notably, MYB target genes are involved in S utilization, while TCP21 ones are involved in photosynthesis (Fig. 6A and Fig. 6B, Table S13).

As mentioned above, our results discovered important changes in gene expression in response to sulfate starvation, suggesting the involvement of different sets of TFs acting in both roots and leaves. However, we found one TF shared by both root and leaf gene regulatory networks which is early induced in roots but has a later response in leaves (Fig. 6C). This TF is member of the EIL family and corresponds to ETHYLENE INSENSITIVE 3-LIKE 3 (EIL3, Solyc01g006650). The closest Arabidopsis gene in terms of amino acid identity is SLIM1, which is a key regulatory factor of the sulfate starvation response [24]. In addition, GO enrichment analysis showed that the identified tomato EIL3 TF might control the expression of a set of genes related to sulfate starvation, including classical sulfate-responsive genes such as LSUs as well as genes encoding enzymes involved in the reductive steps of sulfate assimilation (Table S14). Furthermore, we found that $60 \%$ of the tomato TF target genes are shared with targets predicted for Arabidopsis SLIM1, according to PlantRegMap, including sulfate-response genes such as LSUs and SDIs (Table S14). Overall, we identified several candidate TFs to mediate the transcriptome reprogramming that occurs during sulfate starvation response in roots and leaves, highlighting the possible key regulatory role of the EIL3 TF in tomato.

\section{Discussion}

Temporal and organ-specific transcriptomic response to sulfate starvation in tomato plants

Our current understanding of the transcriptomic response of plants to sulfate availability is mostly based on knowledge gathered in the model plant Arabidopsis thaliana [32]. Most of the analyses carried out in Arabidopsis do not contain whole transcriptome information since they were performed using microarray technology [32, 44]. On the other hand, the tissue-specific response to sulfate availability over time has been poorly explored so far and most studies have been performed at the seedling stage in Arabidopsis. 
Moreover, while transcriptomics approaches have been utilized to uncover the sulfate deficiency response in crops such as wheat [40-43], no transcriptome-wide analysis of the sulfate deficiency response is available for tomato. In this work, we report a comprehensive transcriptome analysis of the sulfate starvation response of tomato plants using RNA-seq, with a temporal and organ resolution.

We found that sulfate starvation has a strong impact on the growth of roots and leaves, starting from 3 weeks after sowing. The inhibition of growth was accompanied with drastic changes in gene expression in both organs. Analysis of enriched GO terms associated with sulfate-responsive genes allowed us to discover several biological processes related to plant growth that were altered by sulfate starvation. To date, several studies have demonstrated that plants activate sulfate uptake by inducing the expression of high-affinity sulfate transporters in response to sulfate starvation [16]. Moreover, it is well established that plants activate the sulfate assimilatory pathway and the degradation of glutathione and other $\mathrm{S}$ compounds such as GSLs during sulfate starvation [16]. As expected, genes involved in sulfate transport and metabolism are differentially expressed according to external sulfate availability in tomato plants, being induced over time in response to sulfate starvation. Specifically, we identified 12 sulfate transporter genes, 3 ATPS and 3 APR genes significantly regulated by sulfate starvation in at least one sample (qvalue $<0.05$ and $\log 2 \mathrm{FC}>1)$ (Table S15).

Our expression analysis also identified several genes involved in cell wall biosynthesis and modification, whose expression was altered by sulfate deprivation. It is well known that cell wall is a crucial component for plant biomass accumulation and cell expansion, which are complementary processes that sustain plant growth [75]. However, how nutrient availability regulates cell wall biosynthesis and organization remains poorly understood [76]. In this work we found that the expression of 6 XET genes decreases over time in sulfate-starved plants (Cluster 1, Table S6). XET genes code for enzymes that have the capacity to loosen cell walls, affecting cell expansion and growth [77]. Thus, repression of XET genes by sulfate starvation can partly explain the observed phenotypes. Accordingly, XET knock-out mutants have reduced cell sizes [78-81], and overexpression or exogenous application of XET proteins decrease cell expansion [82], suggesting that the regulation of XET genes by sulfate starvation might partly explain the observed phenotypes.

Our expression analyses also reveal a significant level of organ-specific response related to the growth inhibition phenotype. Sulfate starvation triggers a strong decrease in the transcript levels of genes related to photosynthesis in leaves, including those related to light and carbon reactions. Taking into account the well-known relationship between plant growth and photosynthesis [83], lower expression of photosynthetic genes likely translates into decreased growth as we observed in the case of sulfate starvation. Interestingly, a similar inhibitory effect of the sulfate starvation on photosynthesis and growth has been demonstrated in other photosynthetic organisms such as microalgae Dunaliella salina [84] and Chlamydomonas reinhardtii [85], suggesting that the regulation of photosynthetic genes by sulfate starvation might be conserved in photosynthetic organisms.

Phosphate levels are regulated by sulfate starvation in tomato

Page $10 / 31$ 
In order to identify novel and specific biological processes in the response to sulfate starvation of tomato plants, we intersected the orthologous groups of genes regulated by sulfate availability in this work with those reported in a previous meta-analysis in Arabidopsis [44]. Remarkably $70 \%$ of the Arabidopsis sulfate-responsive orthologous genes were also regulated by this nutrient in tomato (Fig. 4A), indicating a conserved response to sulfate between Arabidopsis and tomato plants. On the other hand, we also found a group of orthologous genes exclusively regulated by sulfate in tomato plants, which showed overrepresented biological process including "cellular response to phosphate starvation" "photorespiration", "photosynthetic electron transport in photosystem", "ornithine metabolic process", "sucrose metabolic process", among others (Fig. 4C and Table S5), suggesting additional mechanisms might be involved in sulfate starvation responses in tomato.

Remarkably, the most over-represented biological process of orthologous genes regulated by sulfate in tomato plants was "cellular response to phosphate starvation", suggesting that sulfate deficiency affects the internal phosphate levels in tomato plants. In agreement with this result, phosphate content analysis revealed an increase of this nutrient in sulfate-starved plants in both organs. A similar alteration of phosphate levels by sulfate starvation has been reported in Arabidopsis [10,11], with plants showing a 3fold increase of free phosphate in response to S-deficiency. This increase in phosphate levels is not triggered by deficiency of other nutrients such as potassium or iron [10], indicating a specific interaction of sulfate and phosphate homeostasis.

The increase of internal phosphate levels can be achieved by an activation of phosphate transport during sulfate starvation, as has been previously demonstrated in Arabidopsis [11]. Although we found a higher phosphate content in both organs in sulfate-starved plants, phosphate accumulation was higher in leaves, suggesting an increased translocation of phosphate from roots to leaves. In Arabidopsis, phosphate transport from root to shoot is mediated by the PHO1 transporter [86], whose expression is controlled by phosphate starvation at the transcriptional and post-translational levels [9]. PHO1 together with $\mathrm{PHT} 1 ; 9$, a member of the PHT1 family of phosphate transporters, are involved in the increased phosphate translocation from roots to shoots during sulfate starvation in Arabidopsis [11]. We found that several members of the PHO1 and PHT1 gene families (Solyc02g088220, Solyc09g066410 and Solyc09g090080; Figure S10) are induced by sulfate starvation in tomato, suggesting that sulfate starvation promotes phosphate transport from roots to leaves in tomato. These results are consistent with previous works in potato and tomato showing that transcript levels of the phosphate transporters StPT2 and LePT1 increase under sulfate starvation $[87,88]$.

In Arabidopsis, the transcript levels of the main phosphate uptake transporters (PHT1;1, PHT1;2, and $P H T 1 ; 4)$ and $P H O 1$ genes are not affected by sulfate starvation [11], which suggest significant differences in the mechanism of control of phosphate allocation between Arabidopsis and tomato during sulfate starvation. The biological role of increasing phosphate levels in response to sulfate starvation is not fully understood. A rapid replacement of sulfolipids by phospholipids has been reported to occur during the early stage of sulfate starvation [89]. This increased demand of phosphate might require an enhanced acquisition of this nutrient in the later stages of sulfate starvation response. Recent work in 
Arabidopsis has shown that the re-addition of sulfate to the medium restores phosphate levels in shoots and xylem sap [11], indicating a direct relation between sulfate and phosphate levels. However, further research is required to unravel the complex crosstalk between sulfate and phosphate starvation.

\section{Identification of key TFs involved in response to sulfate-starvation in tomato plants}

Gene regulatory network analysis integrating gene expression data and regulatory TF-target data allowed us to identify candidate TFs involved in the response to sulfate starvation during early development of tomato plants. Among the TFs regulated by sulfate starvation in both tissues, we identified a member of the EIL family, whose closest Arabidopsis homolog is SLIM1, a key regulatory factor in the sulfate starvation response [24]. Transcriptome analysis of slim 1 mutants showed lower extent of up-regulation of sulfate transporters and lower extent of down-regulation of GSL genes compared to WT plants [24]. Moreover, SLIM1 is required for plant growth under sulfate starvation conditions [24]. Notably, SLIM1 homologs from rice have been reported to complement the slim 1 mutants from Arabidopsis, indicating a functional conservation of this group of EIL TFs in controlling the sulfate starvation response [24]. Our network analysis highlighted that EIL3 might control an important group of genes involved in sulfate transport and metabolism, like Arabidopsis SLIM1 (Table S14). Specifically, we found that $60 \%$ of the predicted targets of the tomato EIL3 are also described as SLIM1 ones.

Interestingly, SLIM1 transcript or its subcellular localization are not controlled by sulfate deficiency [24], however a recent report shows that SLIM1 can interact with E3 ubiquitin ligases, suggesting SLIM1 ubiquitination and protein degradation might have a role in controlling SLIM1 function in S metabolism [90].In contrast, we found that the transcript levels of tomato EIL3 are dramatically increased by sulfate starvation in roots and leaves of tomato plants. However, we cannot rule out that sulfate starvation might also control EIL3 function at the post-translational level. Further work is therefore required to assess the role of EIL3 in the sulfate response of tomato plants.

Besides EIL3, our analysis identified a Myb TF (Solyc11g071300) as the most connected TF of the root regulatory network. According to the functional analysis of its target genes, the identified Myb TF might be involved in the control of cell division, which is an important biological process for plant growth. In agreement, Arabidopsis orthologues MYB3R-1 and MYB3R-4 act as transcriptional activators regulating cytokinesis [91]. In fact, $53.3 \%$ of the genes from the G2/M-specific class were downregulated in double mutant Arabidopsis plants. Besides, it was uncovered that both MYB3R4 TFs bind to MSA motifs in vitro, which are common cis-elements of G2/M phase-specific genes [92]. All these data suggest that the MYB TFs might play an important role controlling cell division under sulfate starvation conditions.

On the other hand, the most connected TF of the leaf regulatory network was TCP21 (Solyc03g006800). According to the $\mathrm{GO}$ enrichment analysis of its target genes, this TF is related to photosynthesis, which is an essential metabolic process for vegetative growth. Interestingly, several TCP TFs in Arabidopsis have been shown to regulate leaf growth and development [93]. The closest homolog gene of the tomato TCP in Arabidopsis is TCP19, which is involved in the control of leaf senescence in a redundant manner with TCP20 [94]. Our transcriptomic analysis shows that the expression of the identified tomato TCP is 
reduced during sulfate starvation in leaves along with genes related to photosynthesis, leading to a strong reduction in plant growth. Therefore, TCPs might play important functions in the control of leaf growth during sulfate starvation in tomato plants.

\section{Conclusions}

In summary, our results shed lights into the extensive transcriptome reprogramming that is elicited during sulfate starvation in tomato roots and leaves and highlight the role of TFs such as MYB, TCP and EIL3 in coordinating this complex response. We hope this work can serve as a basis for further studies addressing the role of specific genes and processes to further our understanding of the regulatory mechanisms underlying sulfate starvation responses in tomato and other crops.

\section{Methods}

\section{Plant material and growth conditions}

Seeds of the Solanum lycopersicum 'Moneymaker' cultivar (obtained commercially from Semilias, Chile) were grown for 4 weeks in 0.5X Murashige and Skoog (MS) salts [95] (pH 5.7) using rockwool as support material. This liquid culture medium was replaced twice a week to maintain constant nutrient concentrations. For plants grown in sulfate starvation conditions, sulfate salts contained in the MS medium were replaced with equivalent chloride salts [96]. Tomato plants were cultivated in a controlled growth cabinet (Bioref-19, Pitec, Chile) with LED lights $\left(200 \mu \mathrm{mol} \mathrm{m} \mathrm{m}^{-2} \mathrm{~s}^{-1}\right)$ at $22^{\circ} \mathrm{C}$ under 16 -h-light/8-hdark cycles. Three independent experiments were performed using 3 to 5 different plants per replicate.

\section{Plant growth quantification}

For determination of growth-related parameters, leaves and roots were scanned using an Epson Perfection V600 photo scanner. Total leaf area and primary root length were determined from scanned images using the ImageJ software (v1.52) (https://imagej.nih.gov/ij/). Total root and leaf fresh weights were determined using an analytical balance.

\section{Library construction and RNA-Seq data analysis}

Leaves and roots samples were frozen in liquid nitrogen at 2, 3 and 4 weeks after sowing and RNA was isolated using the mirVana miRNA Isolation Kit (Invitrogen), following the manufacturer's instructions for total RNA isolation. The extraction was subjected to on-column DNase I treatment (TURBO DNase, Invitrogen). One microgram of DNase I-treated RNA was used to generate poly-A-enriched sequencing libraries using the TruSeq Stranded mRNA Library Prep kit (Illumina). Libraries were sequenced as 75-nt single-end reads on a NextSeq500 system (Illumina).

The sequenced reads were quality-checked using FastQC version 0.11.5. Taking into account the high quality of the sequenced reads, we skipped the trimming process in order to avoid any potential biases as 
has been previously described [97]. To quantify the mRNA levels of annotated genes, sequenced reads were pseudo-aligned to the publicly available Solanum lycopersicum transcriptome using kallisto (v0.44) [65]. Transcript indices for kallisto were generated from tomato annotation version ITAG3.2 (https://solgenomics.net/organism/Solanum_lycopersicum/genome) which included 35,768 cDNAs. On average, each sample had 30 million reads, of which 27 million reads (90\%) were pseudo-aligned to the tomato transcriptome. The estimated read counts and calculated transcripts per million (TPM) were used for differential expression analysis [66]. Differential expression analysis of the sulfate starvation response in each time and organ was performed using the likelihood ratio test (LRT) implemented in sleuth (v.0.30.0) [66] with three independent replicates. We defined differential gene expression by setting an arbitrary q-value threshold of 0.05 and absolute $\log _{2}$ Fold Change (FC) $>1$.

We performed a multivariate linear model to test whether the expression of a given gene could be explained by the sulfate availability, time or the interaction of both factors using the $\mathrm{R}$ package sleuth (v.0.30.0) [66]. In order to identify significantly regulated genes for each factor, we also applied a q-value threshold of 0.05 and absolute $\log _{2} \mathrm{FC}>1$ in at least one level of each factor. The identification of gene co-expression clusters associated to each factor was performed using the $\mathrm{R}$ package dynamicTreeCut (v.1.63-1) [72], which is a hierarchical clustering method for automatic identification of gene coexpression clusters. Principal component analysis (PCA) was performed on the expression data using pcaExplorer (v.2.14.0) [98].

\section{Gene Ontology enrichment analysis}

Gene Ontology (GO) enrichment analyses were performed using BiNGO (v.3.0.3) [99] with Solanum lycopersicum GO annotations obtained from PLAZA 4.0 [68]. Benjamini and Hochberg false discovery rate (FDR) correction ( $<0.05)$ was applied on the over-represented GO terms obtained in the respective gene set after performing a hypergeometric test with BiNGO. We filtered out the GO terms with less than three annotated genes and used only $\mathrm{GO}$ terms associated to biological processes. To reduce the redundancy between $\mathrm{GO}$ terms, we selected the overrepresented $\mathrm{GO}$ terms with the higher level in the hierarchical GO tree obtained with BiNGO.

\section{Comparative transcriptomics analyses}

In order to determine the sulfate-starvation responsive genes that are shared between Arabidopsis and tomato, we used the ortholog database PLAZA 4.0 [68] to assign a common identification code for the genes of both species (orthologous gene family ID). We intersected both lists using VennPainter software [100] to identify conserved and species-specific sulfate-responsive gene families. The group of Arabidopsis sulfate-responsive genes was obtained from a recent transcriptomic meta-analysis by our group [44]. In the case of tomato, the set of sulfate-responsive genes was obtained from the RNA-seq analysis performed in this work, considering genes significantly regulated by sulfate factor or by the interaction of sulfate and time in roots or leaves (q-value threshold of 0.05 and absolute $\log _{2} F C>1$ ). 
We constructed a gene regulatory network for each organ considering the following criteria: first, we only included in this analysis genes that showed a significant regulation by sulfate or by the interaction of sulfate and time factors (q-value $<0.05$ and absolute $\log _{2} F C>1$ ). Second, we only considered genes that have evidence of TF-target interaction according to the PlantRegMap [74] in order to predict direct regulations. Third, pairs of genes with a TF-target interaction must have a Pearson correlation greater than 0.9 to obtain similar expression profiles between TFs and their target genes. In this manner, we focused on coherent temporal responses of TFs and their targets to sulfate starvation. Pearson correlation was determined from the RNA-seq data obtained in each tissue using the R package rsgcc (v.1.0.6) [101].

Gene regulatory networks were visualized and analyzed using Cytoscape 3.7 [102]. Candidate TFs were selected considering their connectivity and overrepresented GO terms of their target genes.

Quantitative real-time PCR (qPCR)

For qPCR analyses, cDNA was generated from 500ng of total RNA using 5X All-In-One RT MasterMix (Applied Biological Materials, Canada) following the manufacturer's instructions. qPCR reactions were performed with $25 \mathrm{ng}$ of cDNA using the PowerUp SYBR Green Master Mix (Applied Biosystems ${ }^{\mathrm{TM}}$ ) in a CFX96 Real-Time PCR Detection System (Bio-Rad). Raw fluorescent data was analyzed using the Realtime PCR Miner 4.0 software [103] to obtain cycle threshold values and gene amplification efficiencies. The expression of each target gene of interest was normalized by the Actin-7 gene (Solyc11g005330), which showed stable mRNA levels across all samples analyzed by RNA-seq. Sequences of the qPCR primers used in this study are provided in Table S16.

Sulfate and phosphate content analysis

Sulfate content was quantified using a turbidimetric method described by Tabatabai and Bremner [104, 105]. Phosphate content was determined using the Malaquite Green Phosphate Assay Kit (Sigma-Aldrich, Catalog Number MAK307) according to the manufacturer's recommendations.

\section{List Of Abbreviations}

Sulfur (S)

3'-phosphoadenosine 5'-phosphosulfate (PAPS)

High affinity sulfate transporters (SULTRs)

Transcription factors (TFs)

ETHYLENE-INSENSITIVE-LIKE3 (EIL3)/SULFUR LIMITATION 1 (SLIM1)

Glucosinolates (GSLs) 
SULFUR DEFICIENCY 1 (SD1)

LONG HYPOCOTYL 5 (HY5)

MORE SULPHUR ACCUMULATION 1 (MSA1)

Murashige and Skoog salts (MS)

Likelihood ratio test (LRT)

Transcripts per million (TPM)

Fold Change (FC)

Gene Ontology (GO)

False discovery rate (FDR)

Quantitative real-time PCR (qPCR)

Analysis of Variance (ANOVA)

Principal Component Analysis (PCA)

XET (xyloglucan endotransglucosylase/hydrolase)

RESPONSE TO LOW SULFUR (LSU)

Ethylene Insensitive 3-Like3 (EIL3)

\section{Declarations}

Ethics approval and consent to participate: Not applicable

Consent for publication: Not applicable

Availability of data and materials: The RNA-Seq datasets generated and analysed during the current study are available in the NCBI Sequence Read Archive (SRA) repository, accession PRJNA629977, available at https://www.ncbi.nlm.nih.gov/sra/PRJNA629977. All other data generated during this study are included in this published article and its supplementary information files.

Competing interests: The authors declare that they have no competing interests.

Funding: This work was supported by grants from Millennium Institute for Integrative Biology - iBio (Iniciativa Científica Milenio - ANID to E.A.V and J.C), Fondo Nacional de Desarrollo Científico y Tecnológico FONDECYT 1170926 to E.A.V, FONDECYT 1190812 and FONDECYT 1170913 to J.C, 
FONDECYT 3180269 to C.H, PCI-ANID REDES180097 to E.A.V, PCI-ANID REDI170024 to J.C and J.M, The National Institute for Agriculture and Food Research and Technology (INIA) (RTA2015-00014-c02-01 to J.M), UE Prima (PCI2019-103610 to J.M). These funding agencies were not involved in the design of the study, collection, analysis, interpretation of data, and in writing the manuscript.

Authors' contribution: J.C, J.M and E.A.V. conceived the study. J.C, E.A.V, F.U., C.H. and C.L. performed research and analyzed data. J.C, J.M and E.A.V wrote the manuscript. All authors read and approved the final manuscript.

Acknowledgements: We thank Dr. José Miguel Álvarez, from the Center for Genomics and Bioinformatics at Universidad Mayor - iBio for critical reading and helpful comments on the manuscript. We want to acknowledge the "Severo Ochoa Program for Centers of Excellence in R\&D" from the Agencia Estatal de Investigación of Spain (Grant SEV-2016-0672; 2017-2021) for supporting the scientific services used in this work.

\section{References}

1. Kopriva S, Malagoli M, Takahashi H. Sulfur nutrition: impacts on plant development, metabolism, and stress responses. J Exp Bot. 2019;70:4069-73.

2. Nakai Y, Maruyama-Nakashita A. Biosynthesis of Sulfur-Containing Small Biomolecules in Plants. Int J Mol Sci. 2020;21:3470.

3. Takahashi H, Kopriva S, Giordano M, Saito K, Hell R. Sulfur Assimilation in Photosynthetic Organisms: Molecular Functions and Regulations of Transporters and Assimilatory Enzymes. Annu Rev Plant Biol. 2011;62:157-84.

4. Hawkesford M, Horst W, Kichey T, Lambers H, Schjoerring J, Møller IS, et al. Chapter 6 - Functions of Macronutrients. In: Marschner P, editor. Marschner's Mineral Nutrition of Higher Plants (Third Edition). San Diego: Academic Press; 2012. p. 135-89. doi:10.1016/B978-0-12-384905-2.00006-6.

5. Aula L, Dhillon JS, Omara P, Wehmeyer GB, Freeman KW, Raun WR. World Sulfur Use Efficiency for Cereal Crops. Agron J. 2019;111:2485-92.

6. Bouranis DL, Malagoli M, Avice J-C, Bloem E. Advances in Plant Sulfur Research. Plants. 2020;9:256.

7. Raffan S, Oddy J, Halford NG. The Sulphur Response in Wheat Grain and Its Implications for Acrylamide Formation and Food Safety. Int J Mol Sci. 2020;21:3876.

8. White PJ, Bowen HC, Parmaguru P, Fritz M, Spracklen WP, Spiby RE, et al. Interactions between selenium and sulphur nutrition in Arabidopsis thaliana. J Exp Bot. 2004;55:1927-37.

9. Briat JF, Rouached H, Tissot N, Gaymard F, Dubos C. Integration of P, S, Fe, and Zn nutrition signals in Arabidopsis thaliana: Potential involvement of PHOSPHATE STARVATION RESPONSE 1 (PHR1). Front Plant Sci. 2015;6 APR:290.

10. Forieri I, Sticht C, Reichelt M, Gretz N, Hawkesford MJ, Malagoli M, et al. System analysis of metabolism and the transcriptome in Arabidopsis thaliana roots reveals differential co-regulation 
upon iron, sulfur and potassium deficiency. Plant Cell Environ. 2017;40:95-107.

11. Allahham A, Kanno S, Zhang L, Maruyama-Nakashita A. Sulfur Deficiency Increases Phosphate Accumulation, Uptake, and Transport in Arabidopsis thaliana. Int J Mol Sci. 2020;21:2971.

12. Takahashi H. Sulfate transport systems in plants: functional diversity and molecular mechanisms underlying regulatory coordination. J Exp Bot. 2019;70:4075-87.

13. Takahashi H, Yamazaki M, Sasakura N, Watanabe A, Leustek T, Engler J de A, et al. Regulation of sulfur assimilation in higher plants: A sulfate transporter induced in sulfate-starved roots plays a central role in Arabidopsis thaliana. Proc Natl Acad Sci. 1997;94:11102-7.

14. Yoshimoto N, Takahashi H, Smith FW, Yamaya T, Saito K. Two distinct high-affinity sulfate transporters with different inducibilities mediate uptake of sulfate in Arabidopsis roots. Plant J. 2002;29:465-73.

15. Takahashi H. Sulfate transport systems in plants: functional diversity and molecular mechanisms underlying regulatory coordination. J Exp Bot. 2019;70:4075-87.

16. Maruyama-Nakashita A. Metabolic changes sustain the plant life in low-sulfur environments. Curr Opin Plant Biol. 2017;39:144-51.

17. Maruyama-Nakashita A, Nakamura Y, Watanabe-Takahashi A, Inoue E, Yamaya T, Takahashi H. Identification of a novel cis-acting element conferring sulfur deficiency response in Arabidopsis roots. Plant J. 2005;42:305-14.

18. Bielecka M, Watanabe M, Morcuende R, Scheible W-R, Hawkesford MJ, Hesse H, et al. Transcriptome and metabolome analysis of plant sulfate starvation and resupply provides novel information on transcriptional regulation of metabolism associated with sulfur, nitrogen and phosphorus nutritional responses in Arabidopsis. Front Plant Sci. 2015;5. doi:10.3389/fpls.2014.00805.

19. Kumar S, Asif MH, Chakrabarty D, Tripathi RD, Dubey RS, Trivedi PK. Comprehensive analysis of regulatory elements of the promoters of rice sulfate transporter gene family and functional characterization of OsSul1;1 promoter under different metal stress. Plant Signal Behav. 2015;10:e990843.

20. Maruyama-Nakashita A, Watanabe-Takahashi A, Inoue E, Yamaya T, Saito K, Takahashi H. SulfurResponsive Elements in the 3'-Nontranscribed Intergenic Region Are Essential for the Induction of SULFATE TRANSPORTER 2;1 Gene Expression in Arabidopsis Roots under Sulfur Deficiency. Plant Cell. 2015;27:1279-96.

21. Celenza JL, Quiel JA, Smolen GA, Merrikh H, Silvestro AR, Normanly J, et al. The Arabidopsis ATR1 Myb Transcription Factor Controls Indolic Glucosinolate Homeostasis. Plant Physiol. 2005;137:25362.

22. Gigolashvili T, Yatusevich R, Berger B, Müller C, Flügge U-I. The R2R3-MYB transcription factor HAG1/MYB28 is a regulator of methionine-derived glucosinolate biosynthesis in Arabidopsis thaliana. Plant J. 2007;51:247-61.

23. Lee B-R, Koprivova A, Kopriva S. The key enzyme of sulfate assimilation, adenosine 5'phosphosulfate reductase, is regulated by HY5 in Arabidopsis. Plant J. 2011;67:1042-54. 
24. Maruyama-Nakashita A, Nakamura Y, Tohge T, Saito K, Takahashi H. Arabidopsis SLIM1 is a central transcriptional regulator of plant sulfur response and metabolism. Plant Cell. 2006;18:3235-51.

25. Wawrzyńska A, Sirko A. To control and to be controlled: understanding the Arabidopsis SLIM1 function in sulfur deficiency through comprehensive investigation of the EIL protein family. Front Plant Sci. 2014;5. doi:10.3389/fpls.2014.00575.

26. Jones-Rhoades MW, Bartel DP. Computational Identification of Plant MicroRNAs and Their Targets, Including a Stress-Induced miRNA. Mol Cell. 2004;14:787-99.

27. Kawashima CG, Yoshimoto N, Maruyama-Nakashita A, Tsuchiya YN, Saito K, Takahashi H, et al. Sulphur starvation induces the expression of microRNA-395 and one of its target genes but in different cell types. Plant J. 2009;57:313-21.

28. Liang G, Yang F, Yu D. MicroRNA395 mediates regulation of sulfate accumulation and allocation in Arabidopsis thaliana. Plant J. 2010;62:1046-57.

29. Kawashima CG, Matthewman CA, Huang S, Lee B-R, Yoshimoto N, Koprivova A, et al. Interplay of SLIM1 and miR395 in the regulation of sulfate assimilation in Arabidopsis. Plant J. 2011;66:863-76.

30. Matthewman CA, Kawashima CG, Húska D, Csorba T, Dalmay T, Kopriva S. miR395 is a general component of the sulfate assimilation regulatory network in Arabidopsis. FEBS Lett. 2012;586:3242-8.

31. Huang X-Y, Chao D-Y, Koprivova A, Danku J, Wirtz M, Müller S, et al. Nuclear Localised MORE SULPHUR ACCUMULATION1 Epigenetically Regulates Sulphur Homeostasis in Arabidopsis thaliana. PLoS Genet. 2016;12. doi:10.1371/journal.pgen.1006298.

32. Watanabe M, Hoefgen R. Sulphur systems biology-making sense of omics data. J Exp Bot. 2019;70:4155-70.

33. Nikiforova V, Freitag J, Kempa S, Adamik M, Hesse H, Hoefgen R. Transcriptome analysis of sulfur depletion in Arabidopsis thaliana: interlacing of biosynthetic pathways provides response specificity. Plant J. 2003;33:633-50.

34. Hirai MY, Fujiwara T, Awazuhara M, Kimura T, Noji M, Saito K. Global expression profiling of sulfurstarved Arabidopsis by DNA macroarray reveals the role of 0-acetyl-I-serine as a general regulator of gene expression in response to sulfur nutrition. Plant J. 2003;33:651-63.

35. Maruyama-Nakashita A, Inoue E, Watanabe-Takahashi A, Yamaya T, Takahashi H. Transcriptome Profiling of Sulfur-Responsive Genes in Arabidopsis Reveals Global Effects of Sulfur Nutrition on Multiple Metabolic Pathways. Plant Physiol. 2003;132:597-605.

36. Higashi Y, Hirai MY, Fujiwara T, Naito S, Noji M, Saito K. Proteomic and transcriptomic analysis of Arabidopsis seeds: molecular evidence for successive processing of seed proteins and its implication in the stress response to sulfur nutrition. Plant J. 2006;48:557-71.

37. Iyer-Pascuzzi AS, Jackson T, Cui H, Petricka JJ, Busch W, Tsukagoshi H, et al. Cell Identity Regulators Link Development and Stress Responses in the Arabidopsis Root. Dev Cell. 2011;21:770-82.

38. Aarabi F, Kusajima M, Tohge T, Konishi T, Gigolashvili T, Takamune M, et al. Sulfur deficiencyinduced repressor proteins optimize glucosinolate biosynthesis in plants. Sci Adv. 2016;2:e1601087. 
39. Dong Y, Silbermann M, Speiser A, Forieri I, Linster E, Poschet G, et al. Sulfur availability regulates plant growth via glucose-TOR signaling. Nat Commun. 2017;8:1-10.

40. Howarth JR, Parmar S, Jones J, Shepherd CE, Corol D-I, Galster AM, et al. Co-ordinated expression of amino acid metabolism in response to $\mathrm{N}$ and $\mathrm{S}$ deficiency during wheat grain filling. J Exp Bot. 2008;59:3675-89.

41. Gupta S, Yadav BS, Raj U, Freilich S, Varadwaj PK. Transcriptomic Analysis of Soil Grown T. aestivum cv. Root to Reveal the Changes in Expression of Genes in Response to Multiple Nutrients Deficiency. Front Plant Sci. 2017;8. doi:10.3389/fpls.2017.01025.

42. Yu Z, Juhasz A, Islam S, Diepeveen D, Zhang J, Wang P, et al. Impact of mid-season sulphur deficiency on wheat nitrogen metabolism and biosynthesis of grain protein. Sci Rep. 2018;8:2499.

43. Dai Z, Plessis A, Vincent J, Duchateau N, Besson A, Dardevet M, et al. Transcriptional and metabolic alternations rebalance wheat grain storage protein accumulation under variable nitrogen and sulfur supply. Plant J. 2015;83:326-43.

44. Henríquez-Valencia C, Arenas-M A, Medina J, Canales J. Integrative Transcriptomic Analysis Uncovers Novel Gene Modules That Underlie the Sulfate Response in Arabidopsis thaliana. Front Plant Sci. 2018.

45. Hirai MY, Yano M, Goodenowe DB, Kanaya S, Kimura T, Awazuhara M, et al. Integration of transcriptomics and metabolomics for understanding of global responses to nutritional stresses in Arabidopsis thaliana. Proc Natl Acad Sci. 2004;101:10205-10.

46. Hirai MY, Klein M, Fujikawa Y, Yano M, Goodenowe DB, Yamazaki Y, et al. Elucidation of gene-to-gene and metabolite-to-gene networks in arabidopsis by integration of metabolomics and transcriptomics. J Biol Chem. 2005;280:25590-5.

47. Zhang J, Sun X, Zhang Z, Ni Y, Zhang Q, Liang X, et al. Metabolite profiling of Arabidopsis seedlings in response to exogenous sinalbin and sulfur deficiency. Phytochemistry. 2011;72:1767-78.

48. Sung J, Baek S, Kim J, X. Kim Y, Lee Y, Lee S, et al. Responses of Primary Metabolites and Glucosinolates in Sulfur Deficient-Cabbage (Brassica rapa L. ssp. Pekinensis). J Plant Biochem Physiol. 2018;06. doi:10.4172/2329-9029.1000223.

49. Ghosson H, Schwarzenberg A, Jamois F, Yvin J-C. Simultaneous untargeted and targeted metabolomics profiling of underivatized primary metabolites in sulfur-deficient barley by ultra-high performance liquid chromatography-quadrupole/time-of-flight mass spectrometry. Plant Methods. 2018;14:62.

50. D’Hooghe P, Escamez S, Trouverie J, Avice J-C. Sulphur limitation provokes physiological and leaf proteome changes in oilseed rape that lead to perturbation of sulphur, carbon and oxidative metabolisms. BMC Plant Biol. 2013;13:23.

51. D’Hooghe P, Dubousset L, Gallardo K, Kopriva S, Avice J-C, Trouverie J. Evidence for proteomic and metabolic adaptations associated with alterations of seed yield and quality in sulfur-limited Brassica napus L. Mol Cell Proteomics. 2014;13:1165-83. 
52. Yıldız M, Terzi H. Proteomic analysis of chromium stress and sulfur deficiency responses in leaves of two canola (Brassica napus L.) cultivars differing in $\mathrm{Cr}(\mathrm{VI})$ tolerance. Ecotoxicol Environ Saf. 2016;124:255-66.

53. Bagheri R, Ahmad J, Bashir H, Iqbal M, Qureshi Ml. Changes in rubisco, cysteine-rich proteins and antioxidant system of spinach (Spinacia oleracea L.) due to sulphur deficiency, cadmium stress and their combination. Protoplasma. 2017;254:1031-43.

54. Sønderby IE, Hansen BG, Bjarnholt N, Ticconi C, Halkier BA, Kliebenstein DJ. A systems biology approach identifies a R2R3 MYB gene subfamily with distinct and overlapping functions in regulation of aliphatic glucosinolates. PloS One. 2007;2:e1322.

55. Nikiforova VJ, Daub CO, Hesse H, Willmitzer L, Hoefgen R. Integrative gene-metabolite network with implemented causality deciphers informational fluxes of sulphur stress response. J Exp Bot. 2005;56:1887-96.

56. Falkenberg B, Witt I, Zanor MI, Steinhauser D, Mueller-Roeber B, Hesse H, et al. Transcription factors relevant to auxin signalling coordinate broad-spectrum metabolic shifts including sulphur metabolism. J Exp Bot. 2008;59:2831-46.

57. Kimura S, Sinha N. Tomato (Solanum lycopersicum): A Model Fruit-Bearing Crop. Cold Spring Harb Protoc. 2008;2008.

58. Canene-Adams K, Campbell JK, Zaripheh S, Jeffery EH, Erdman JW. The Tomato As a Functional Food. J Nutr. 2005;135:1226-30.

59. Alhendawi RA, Kirkby EA, Pilbeam DJ. Evidence That Sulfur Deficiency Enhances Molybdenum Transport in Xylem Sap of Tomato Plants. J Plant Nutr. 2005;28:1347-53.

60. Lopez J, Tremblay N, Voogt W, Dubé S, Gosselin A. Effects of varying sulphate concentrations on growth, physiology and yield of the greenhouse tomato. Sci Hortic. 1996;67:207-17.

61. Hasan MdK, Liu C-X, Pan Y-T, Ahammed GJ, Qi Z-Y, Zhou J. Melatonin alleviates low-sulfur stress by promoting sulfur homeostasis in tomato plants. Sci Rep. 2018;8:10182.

62. Zuchi S, Cesco S, Varanini Z, Pinton R, Astolfi S. Sulphur deprivation limits Fe-deficiency responses in tomato plants. Planta. 2009;230:85-94.

63. Zuchi S, Watanabe M, Hubberten H-M, Bromke M, Osorio S, Fernie AR, et al. The Interplay between Sulfur and Iron Nutrition in Tomato. Plant Physiol. 2015;169:2624.

64. Cerdá A, Martínez V, Caro M, Fernández FG. Effect of sulfur deficiency and excess on yield and sulfur accumulation in tomato plants. J Plant Nutr. 1984;7:1529-43.

65. Bray NL, Pimentel H, Melsted P, Pachter L. Near-optimal probabilistic RNA-seq quantification. Nat Biotechnol. 2016;34:525-7.

66. Pimentel H, Bray NL, Puente S, Melsted P, Pachter L. Differential analysis of RNA-seq incorporating quantification uncertainty. Nat Methods. 2017;14:687-90.

67. Li L, Stoeckert CJ, Roos DS. OrthoMCL: Identification of Ortholog Groups for Eukaryotic Genomes. Genome Res. 2003;13:2178-89. 
68. Aleksza D, Horváth GV, Sándor G, Szabados L, Barragan V, Leidi EO, et al. PLAZA 4.0: An integrative resource for functional, evolutionary and comparative plant genomics. Nucleic Acids Res. 2017.

69. Duan K, Yi K, Dang L, Huang H, Wu W, Wu P. Characterization of a sub-family of Arabidopsis genes with the SPX domain reveals their diverse functions in plant tolerance to phosphorus starvation. Plant J. 2008;54:965-75.

70. Okazaki Y, Otsuki H, Narisawa T, Kobayashi M, Sawai S, Kamide Y, et al. A new class of plant lipid is essential for protection against phosphorus depletion. Nat Commun. 2013;4.

71. Sun Y, Jain A, Xue Y, Wang X, Zhao G, Liu L, et al. OsSQD1 at the crossroads of phosphate and sulfur metabolism affects plant morphology and lipid composition in response to phosphate deprivation. Plant Cell Environ. 2020;43:1669-90.

72. Langfelder $P$, Zhang $B$, Horvath S. Defining clusters from a hierarchical cluster tree: the Dynamic Tree Cut package for R. Bioinformatics. 2008;24:719-20.

73. Schwacke R, Ponce-Soto GY, Krause K, Bolger AM, Arsova B, Hallab A, et al. MapMan4: A Refined Protein Classification and Annotation Framework Applicable to Multi-Omics Data Analysis. Mol Plant. 2019;12:879-92.

74. Tian F, Yang D-C, Meng Y-Q, Jin J, Gao G. PlantRegMap: charting functional regulatory maps in plants. Nucleic Acids Res. 2020;48:D1104-13.

75. Ivakov A, Flis A, Apelt F, Fünfgeld M, Scherer U, Stitt M, et al. Cellulose synthesis and cell expansion are regulated by different mechanisms in growing arabidopsis hypocotyls. Plant Cell. 2017;29:130515.

76. Ogden M, Hoefgen R, Roessner U, Persson S, Khan G. Feeding the Walls: How Does Nutrient Availability Regulate Cell Wall Composition? Int J Mol Sci. 2018;19:2691.

77. Miedes E, Suslov D, Vandenbussche F, Kenobi K, Ivakov A, Van Der Straeten D, et al. Xyloglucan endotransglucosylase/hydrolase (XTH) overexpression affects growth and cell wall mechanics in etiolated Arabidopsis hypocotyls. J Exp Bot. 2013;64:2481-97.

78. Osato Y, Yokoyama R, Nishitani K. A principal role for AtXTH18 in Arabidopsis thaliana root growth: a functional analysis using RNAi plants. J Plant Res. 2006;119:153-62.

79. Liu Y-B, Lu S-M, Zhang J-F, Liu S, Lu Y-T. A xyloglucan endotransglucosylase/hydrolase involves in growth of primary root and alters the deposition of cellulose in Arabidopsis. Planta. 2007;226:154760.

80. Sasidharan R, Chinnappa CC, Staal M, Elzenga JTM, Yokoyama R, Nishitani K, et al. Light QualityMediated Petiole Elongation in Arabidopsis during Shade Avoidance Involves Cell Wall Modification by Xyloglucan Endotransglucosylase/Hydrolases. Plant Physiol. 2010;154:978.

81. Ohba T, Takahashi S, Asada K. Alteration of fruit characteristics in transgenic tomatoes with modified expression of a xyloglucan endotransglucosylase/hydrolase gene. Plant Biotechnol. 2011;28:25-32.

82. Maris A, Suslov D, Fry SC, Verbelen J-P, Vissenberg K. Enzymic characterization of two recombinant xyloglucan endotransglucosylase/hydrolase (XTH) proteins of Arabidopsis and their effect on root 
growth and cell wall extension. J Exp Bot. 2009;60:3959-72.

83. Kirschbaum MUF. Does enhanced photosynthesis enhance growth? Lessons learned from $\mathrm{CO} 2$ enrichment studies. Plant Physiol. 2011;155:117-24.

84. Giordano M, Pezzoni V, Hell R. Strategies for the allocation of resources under sulfur limitation in the green alga Dunaliella salina. Plant Physiol. 2000;124:857-64.

85. Davies JP, Yildiz FH, Grossman A. Sac1, a putative regulator that is critical for survival of Chlamydomonas reinhardtii during sulfur deprivation. EMBO J. 1996;15:2150-9.

86. Hamburger D, Rezzonico E, Petétot JMDC, Somerville C, Poirier Y. Identification and characterization of the Arabidopsis PHO1 gene involved in phosphate loading to the xylem. Plant Cell. 2002;14:889902.

87. Leggewie G, Willmitzer L, Riesmeier JW. Two cDNAs from potato are able to complement a phosphate uptake-deficient yeast mutant: identification of phosphate transporters from higher plants. Plant Cell. 1997;9:381-92.

88. Daram P, Brunner S, Persson BL, Amrhein N, Bucher M. Functional analysis and cell-specific expression of a phosphate transporter from tomato. Planta. 1998;206:225-33.

89. Rouached H. Multilevel coordination of phosphate and sulfate homeostasis in plants. Plant Signal Behav. 2011;6:952-5.

90. Wawrzyńska A, Sirko A. Proteasomal Degradation of Proteins is Important for the Proper Transcriptional Response to Sulfur Deficiency Conditions in Plants. Plant Cell Physiol. 2020. doi:10.1093/pcp/pcaa076.

91. Haga N, Kobayashi K, Suzuki T, Maeo K, Kubo M, Ohtani M, et al. Mutations in MYB3R1 and MYB3R4 cause pleiotropic developmental defects and preferential down-regulation of multiple G2/M-specific genes in Arabidopsis. Plant Physiol. 2011;157:706-17.

92. Ito $M$, Iwase $M$, Kodama $H$, Lavisse $P$, Komamine A, Nishihama $R$, et al. A novel cis-acting element in promoters of plant B-type cyclin genes activates M phase-specific transcription. Plant Cell. 1998;10:331-41.

93. Danisman S. TCP transcription factors at the interface between environmental challenges and the plant's growth responses. Front Plant Sci. 2016;7:1930.

94. Danisman S, van Dijk ADJ, Bimbo A, van der Wal F, Hennig L, de Folter $S$, et al. Analysis of functional redundancies within the Arabidopsis TCP transcription factor family. J Exp Bot. 2013;64:5673-85.

95. Murashige T, Skoog F. A Revised Medium for Rapid Growth and Bio Assays with Tobacco Tissue Cultures. Physiol Plant. 1962;15:473-97.

96. Yoshimoto N, Inoue E, Watanabe-Takahashi A, Saito K, Takahashi H. Posttranscriptional Regulation of High-Affinity Sulfate Transporters in Arabidopsis by Sulfur Nutrition. Plant Physiol. 2007;145:37888.

97. Williams CR, Baccarella A, Parrish JZ, Kim CC. Trimming of sequence reads alters RNA-Seq gene expression estimates. BMC Bioinformatics. 2016;17. doi:10.1186/s12859-016-0956-2. 
98. Marini F, Binder H. pcaExplorer: an R/Bioconductor package for interacting with RNA-seq principal components. BMC Bioinformatics. 2019;20:331.

99. Maere S, Heymans K, Kuiper M. BiNGO: a Cytoscape plugin to assess overrepresentation of gene ontology categories in biological networks. Bioinformatics. 2005;21:3448-9.

100. Lin G, Chai J, Yuan S, Mai C, Cai L, Murphy RW, et al. VennPainter: A Tool for the Comparison and Identification of Candidate Genes Based on Venn Diagrams. PloS One. 2016;11:e0154315.

101. Ma C, Wang X. Application of the Gini correlation coefficient to infer regulatory relationships in transcriptome analysis. Plant Physiol. 2012;160:192-203.

102. Shannon P, Markiel A, Ozier O, Baliga NS, Wang JT, Ramage D, et al. Cytoscape: A Software Environment for Integrated Models of Biomolecular Interaction Networks. Genome Res. 2003;13:2498-504.

103. Zhao S, Fernald RD. Comprehensive algorithm for quantitative real-time polymerase chain reaction. J Comput Biol J Comput Mol Cell Biol. 2005;12:1047-64.

104. Tabatabai MA, Bremner JM. A Simple Turbidimetric Method of Determining Total Sulfur in Plant Materials1. Agron J. 1970;62:805-6.

105. Kanno S, Cuyas L, Javot H, Bligny R, Gout E, Dartevelle T, et al. Performance and Limitations of Phosphate Quantification: Guidelines for Plant Biologists. Plant Cell Physiol. 2016;57:690-706.

\section{Figures}




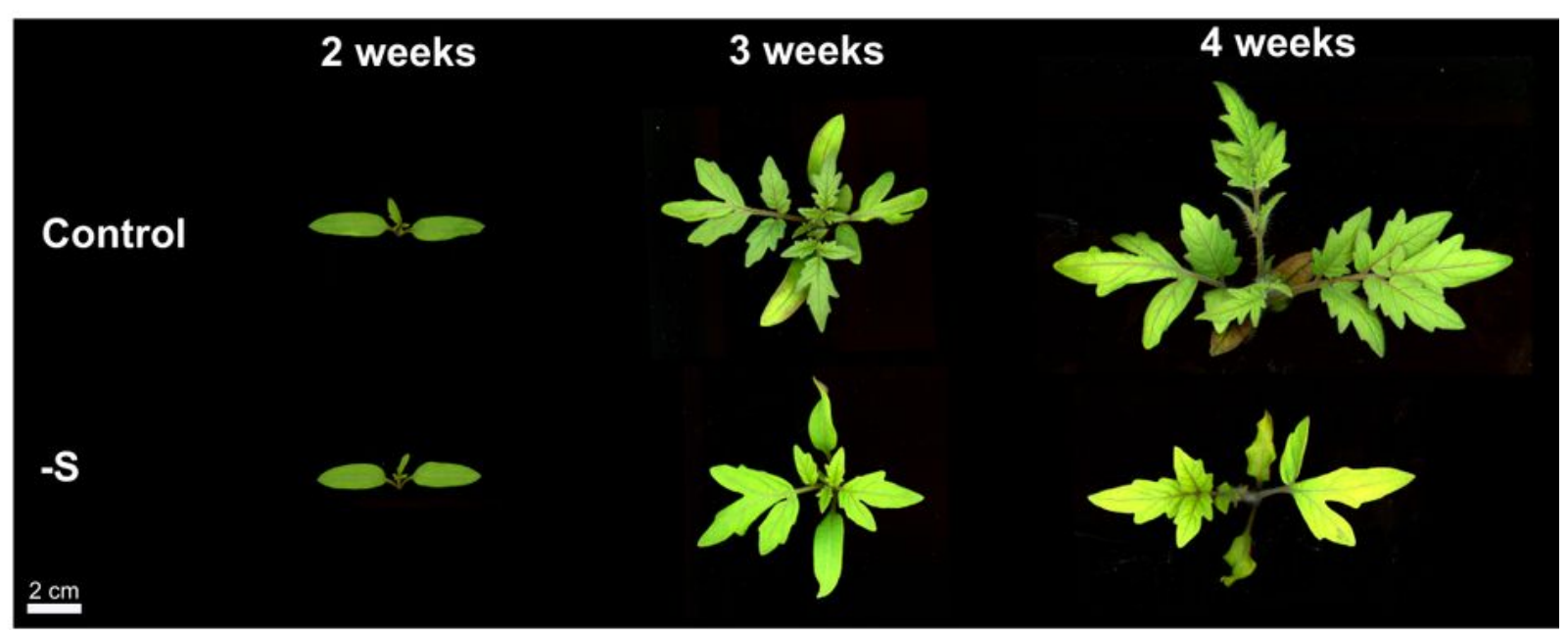

B

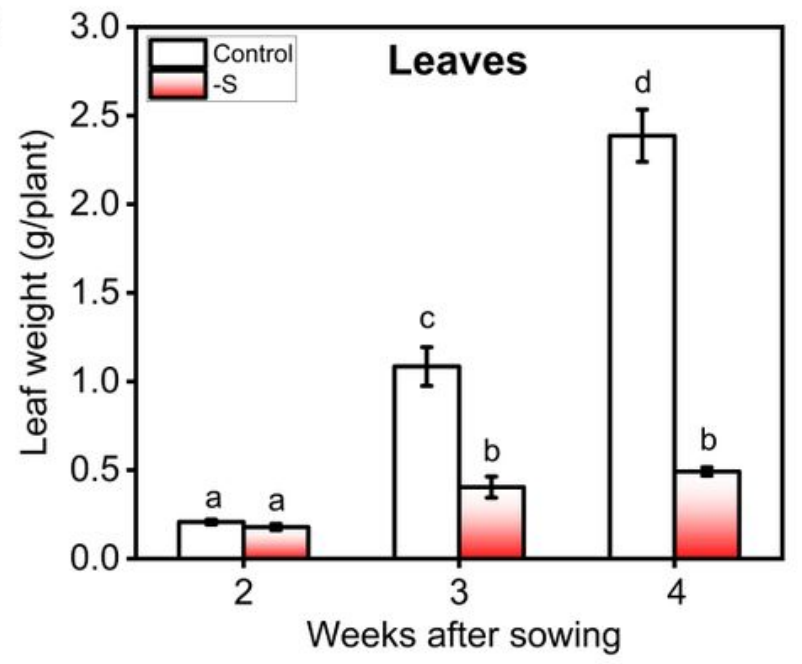

D

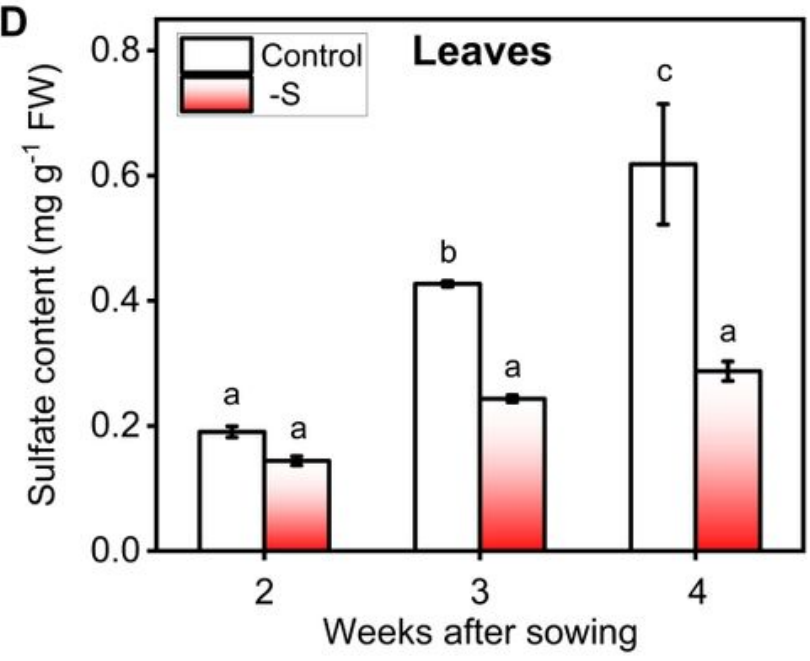

C
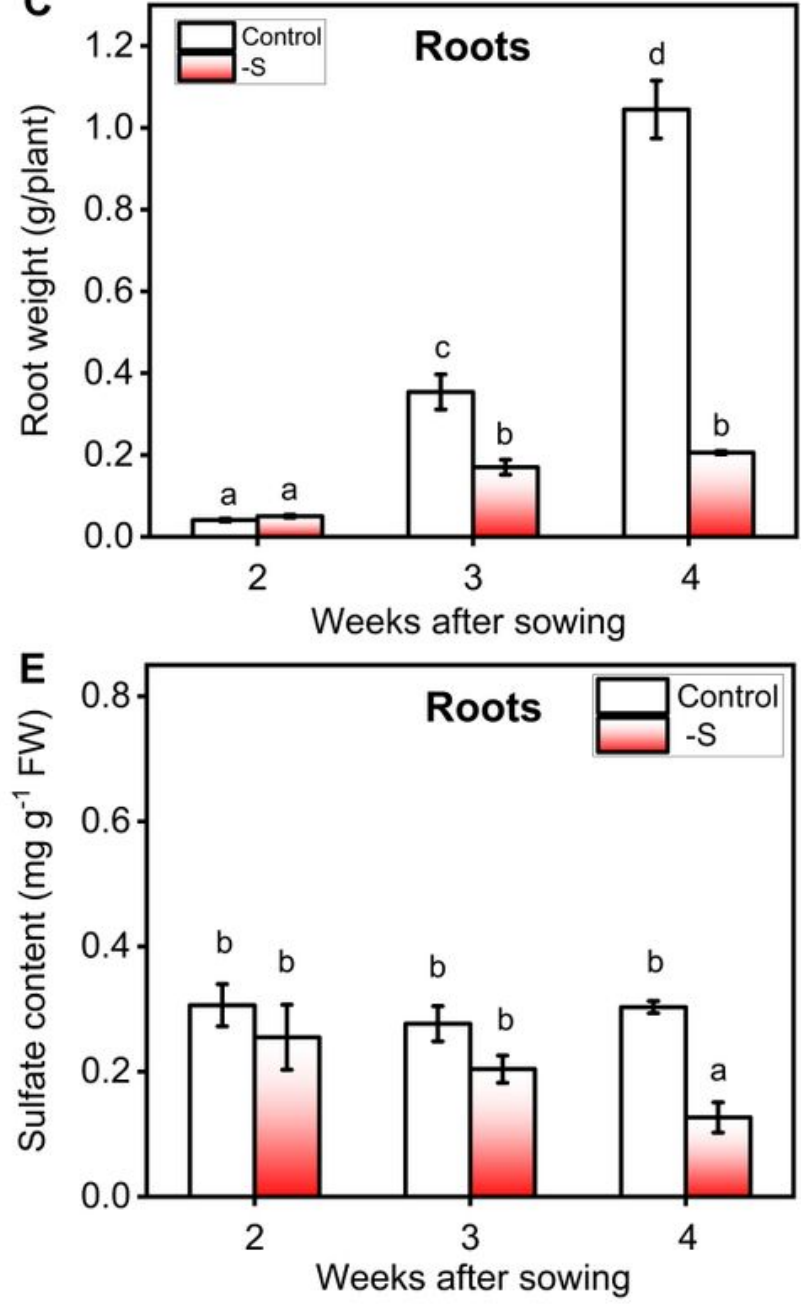

\section{Figure 1}

External sulfate availability is required for optimal plant growth. A) Representative images of tomato plants (Solanum lycopersicum cv. Moneymaker) grown under full nutrient or S-limiting conditions for 4 weeks. Biomass accumulation is reduced by sulfate deficiency from 3 weeks after sowing. Fresh weight for leaves (B) and roots (C) were measured from 2 to 4 weeks after sowing. Sulfate content of leaves (D) and roots (E) were determined from 2 to 4 weeks after sowing using the turbidimetric method [23]. Values 
plotted correspond to the means of three independent experiments \pm the standard error of the mean.

Means with different letters indicate significant differences ( $P<0.05$ two-way ANOVA and Tukey's test). 35 different plants were measured for each experimental replicate.
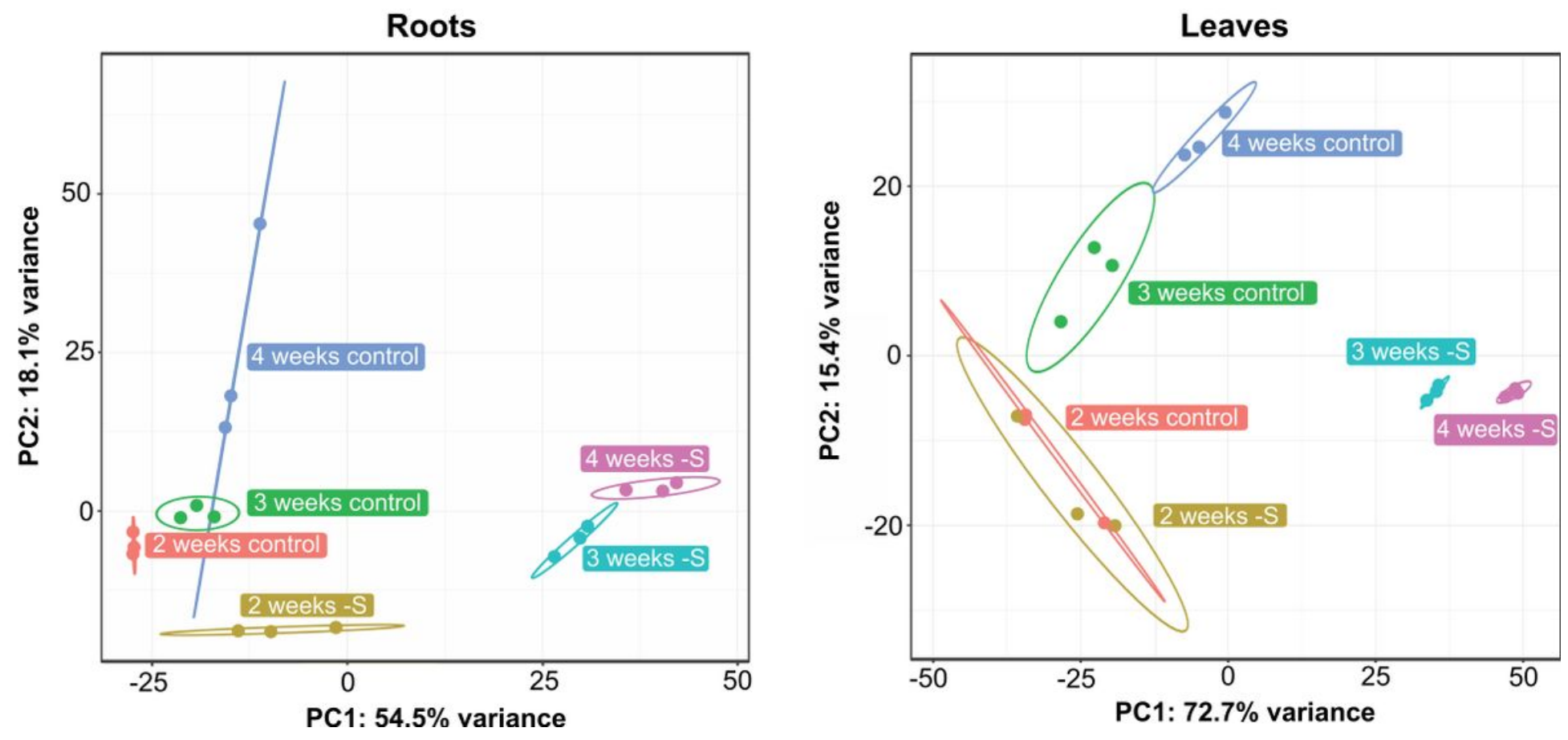

Figure 2

Exploratory data analysis of tomato RNA-seq shows that the response to sulfate starvation begins in root tissue. Principal Component Analysis (PCA) of RNA-seq data of roots (left) and leaves (right) samples. PCA analysis was performed with pcaExplorer R package [65] using log2-transformed normalized expression data. Ellipses represents the $95 \%$ confidence interval of 3 independent experiments. Replicates of the same experiment are indicated with the same color.

A

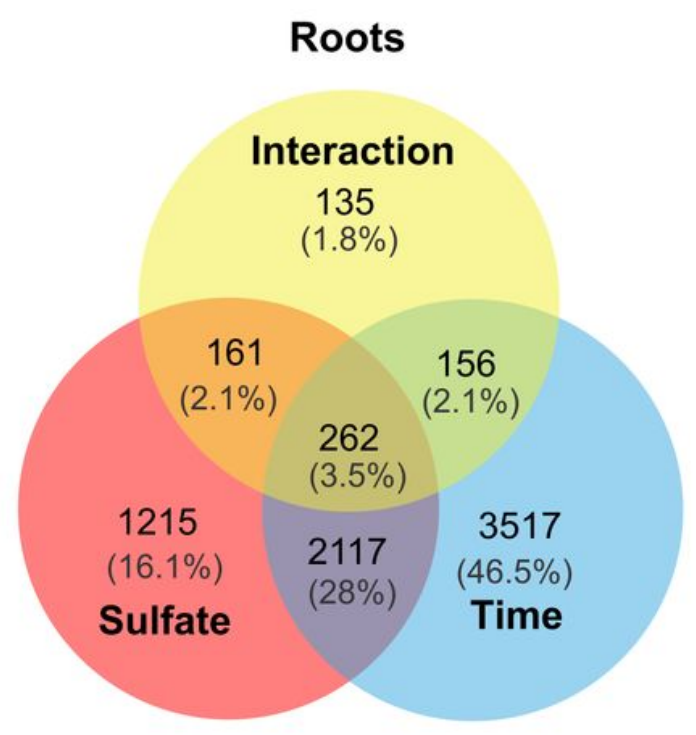

B

\section{Leaves}

Interaction

392

(4.9\%)

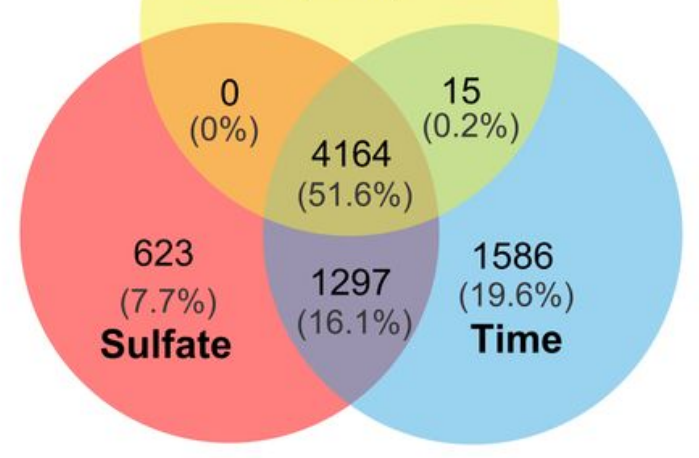

Figure 3 
The impact of sulfate starvation on the transcriptome depends on the age of the plants in leaves but not in roots. Venn diagram showing genes significantly regulated by sulfate, time or by interaction of both factors in roots (A) and leaves (B). Multifactorial analysis was performed using sleuth $R$ package [32] with a q-value $<0.05$.

A

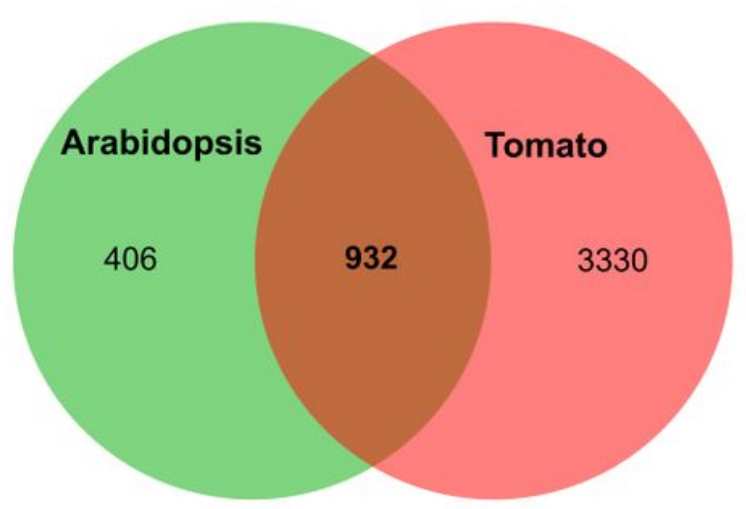

C

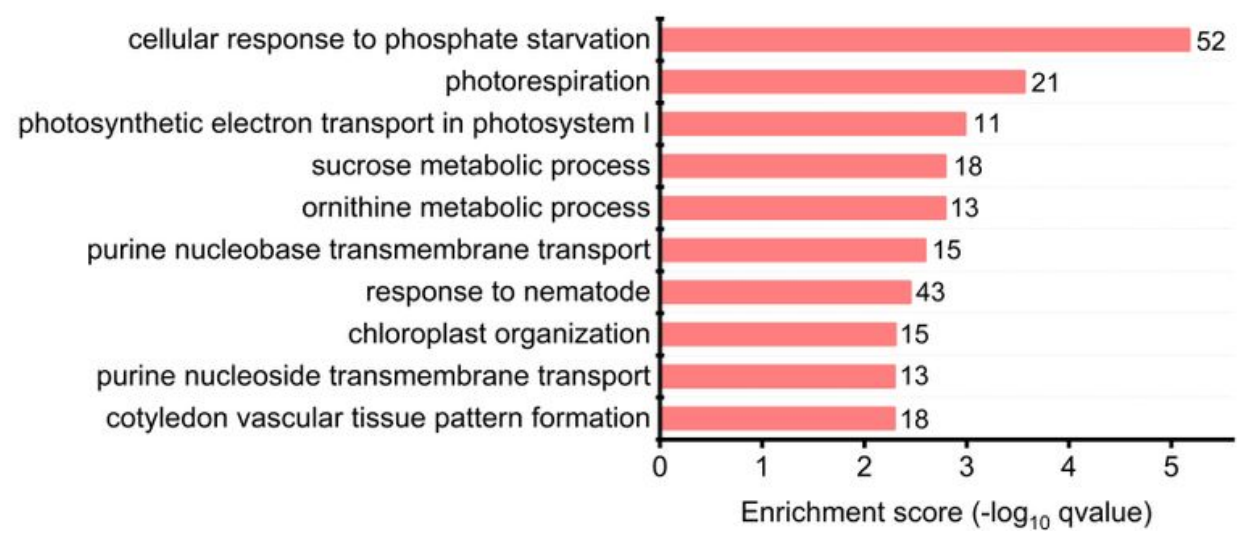

B

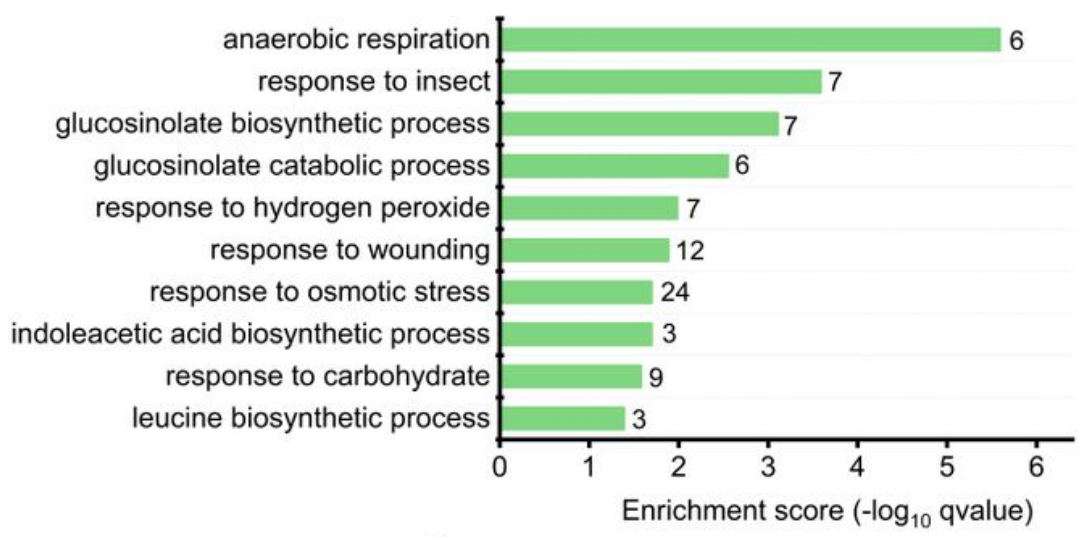

D

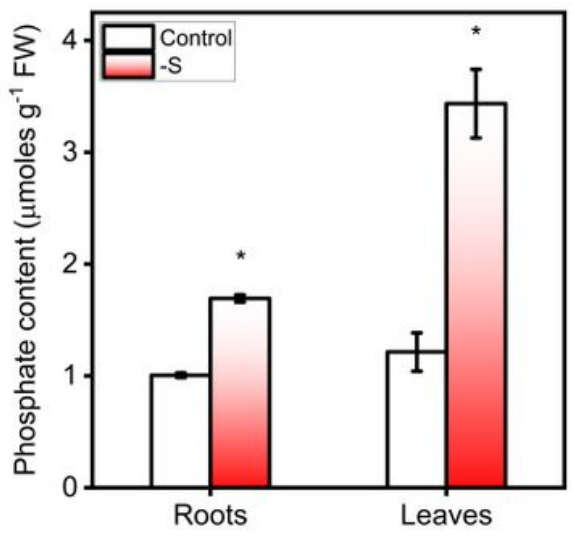

\section{Figure 4}

Comparative transcriptome analysis of Arabidopsis and tomato plants in response to sulfate starvation reveal conserved and specific features. A) Venn diagram showing sulfate-responsive genes shared between Arabidopsis and tomato plants. The plant comparative genomics resource PLAZA 4.0 was used to identify the orthologous genes. The Arabidopsis sulfate-responsive genes were obtained from a metaanalysis [15]. B) GO term enrichment analysis of genes exclusively regulated by sulfate in Arabidopsis. The 10 most over-represented biological functions are shown along with the number of genes belongs to each GO term. C) GO term enrichment analysis of genes exclusively regulated by sulfate in tomato plants. The 10 most over-represented biological functions are shown along with the number of genes belongs to each GO term. D) Sulfate starvation increase phosphate content in tomato roots and leaves of plants grown under full nutrient or S-limiting conditions for 3 weeks. Phosphate content was determined by the malachite green assay [72]. A Student's t-test was performed to test the significance $(P<0.05)$ of the differences between sulfate-starved and control samples $\left({ }^{*}\right)$. Values plotted correspond to the means of three independent experiments \pm standard deviation. 3-5 different plants were measured for each experimental replicate. 


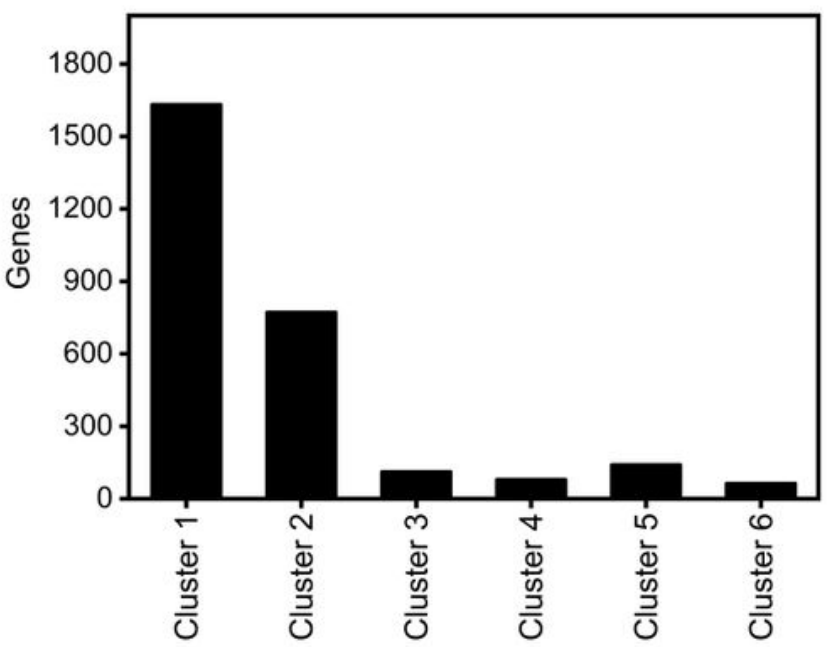

B

Leaves

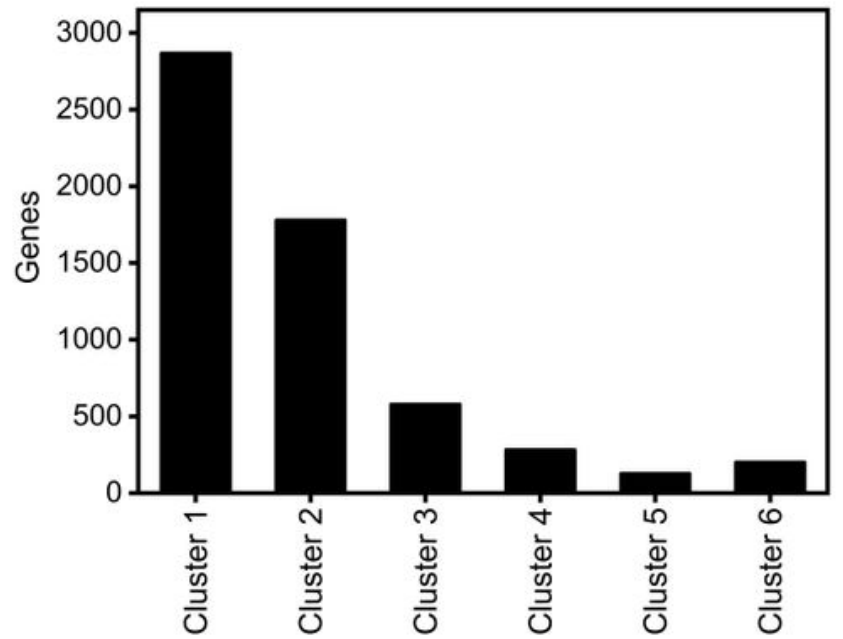

C

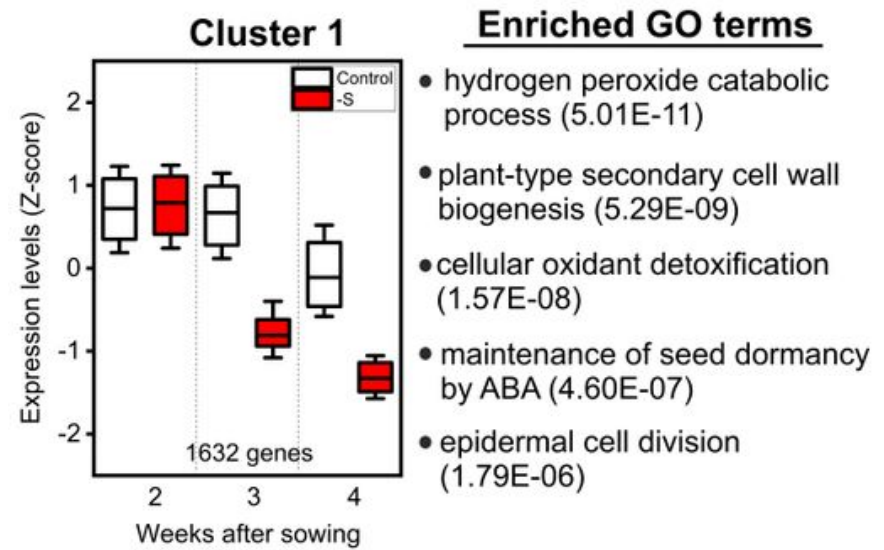

Roots

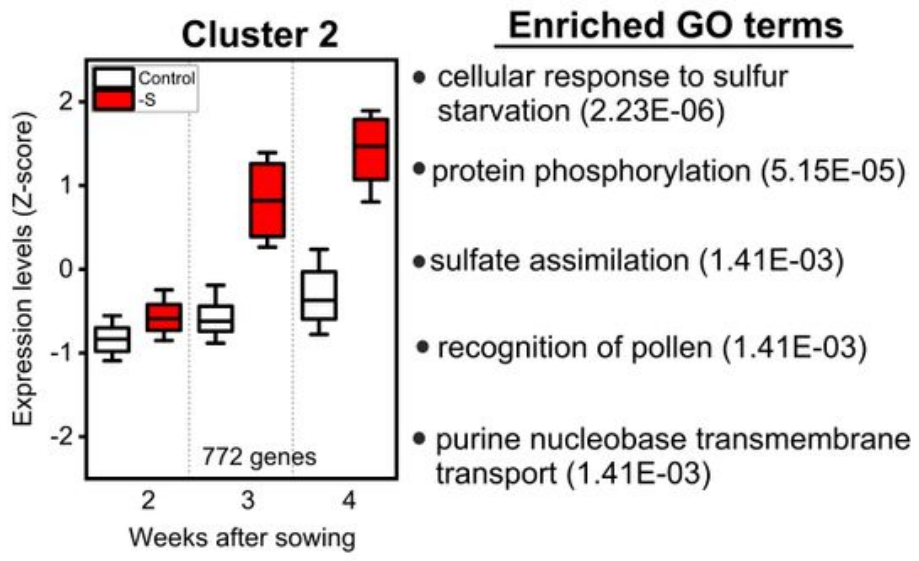

D

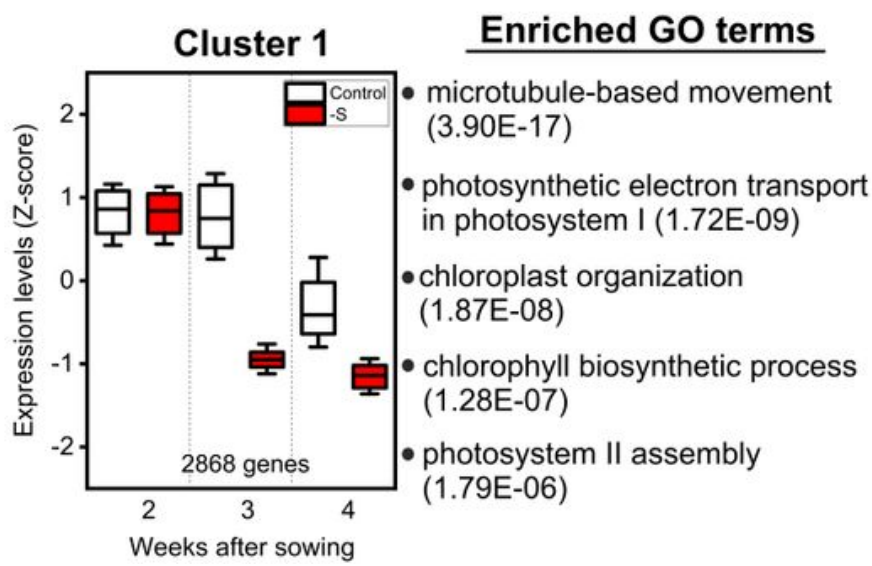

Leaves

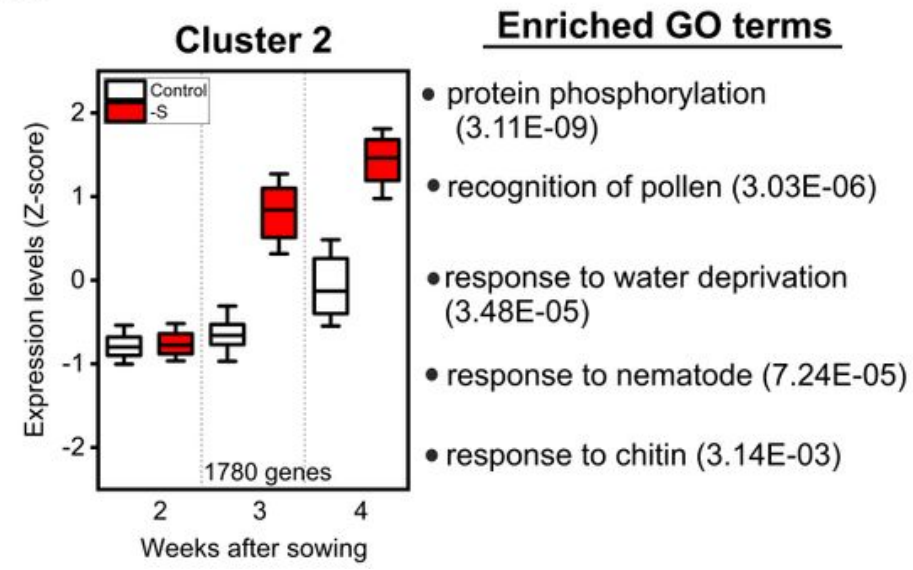

\section{Figure 5}

Gene co-expression clusters of roots and leaves during tomato development under sulfate starvation. Coexpression clusters of genes exclusively regulated by sulfate and time in roots (A) or leaves (B). Expression patters of major clusters in roots (C) and leaves (D). On each box, the central mark indicates the median, and the bottom and top edges of the box indicate the 25th and 75th percentiles, respectively. 
Whisker indicates standard deviation of the expression data of all genes belongs to the cluster. The top 5 enriched GO terms are indicated to the right of each box plot with the q-values in brackets.

A

Root regulatory network

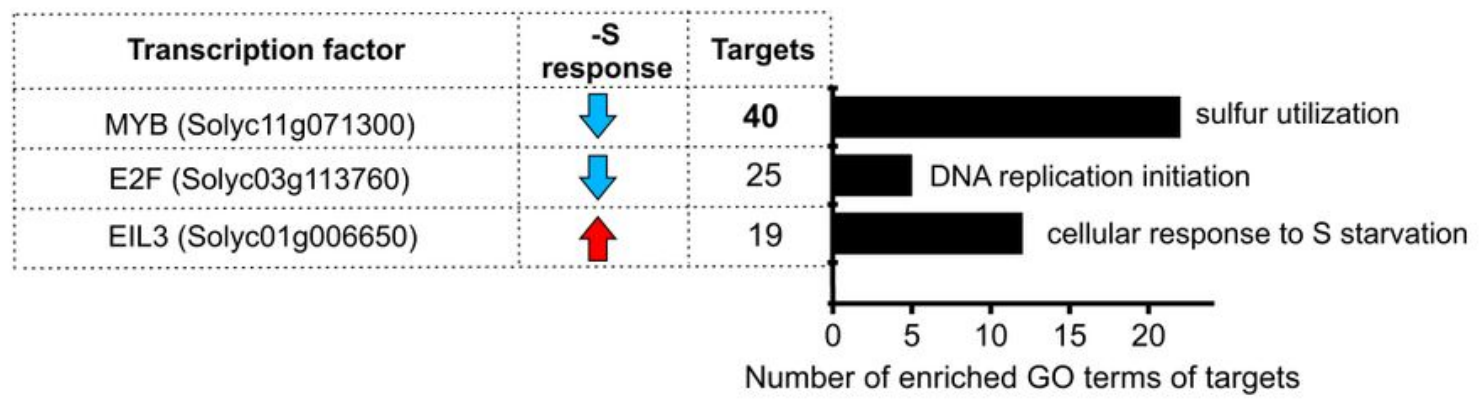

B

Leaf regulatory network

\begin{tabular}{|c|c|c|c|}
\hline Transcription factor & $\stackrel{-S}{\text { response }}$ & Targets & \\
\hline TCP21 (Solyc03g006800) & & 71 & chlorophyll biosynthetic process \\
\hline bHLH36 (Solyc05g006650) & & 54 & regulation of auxin biosynthetic process \\
\hline GAGA-binding (Solyc04g081170) & & 46 & gibberellic acid mediated signaling pathway \\
\hline Zinc finger (Solyc02g085580) & & 43 & gibberellic acid mediated signaling pathway \\
\hline WRKY71 (Solyc02g071130) & & 26 & cellular response to antibiotic \\
\hline MYB60 (Solyc10g081490) & & 21 & cutin biosynthetic process \\
\hline WRKY6 (Solyc02g080890) & & 18 & response to boron-containing substance \\
\hline NAC (Solyc07g045030) & & 18 & leaf senescence \\
\hline EIL3 (Solyc01g006650) & & 17 & cellular response to S starvation \\
\hline \multirow[t]{3}{*}{ NAC (Solyc01g009860) } & & 11 & positive regulation of leaf senescence \\
\hline & & & $\begin{array}{llll}5 & 10 & 15 & 20\end{array}$ \\
\hline & & Numb & of enriched GO terms of targets \\
\hline
\end{tabular}

C

EIL3 (Solyc01g006650)
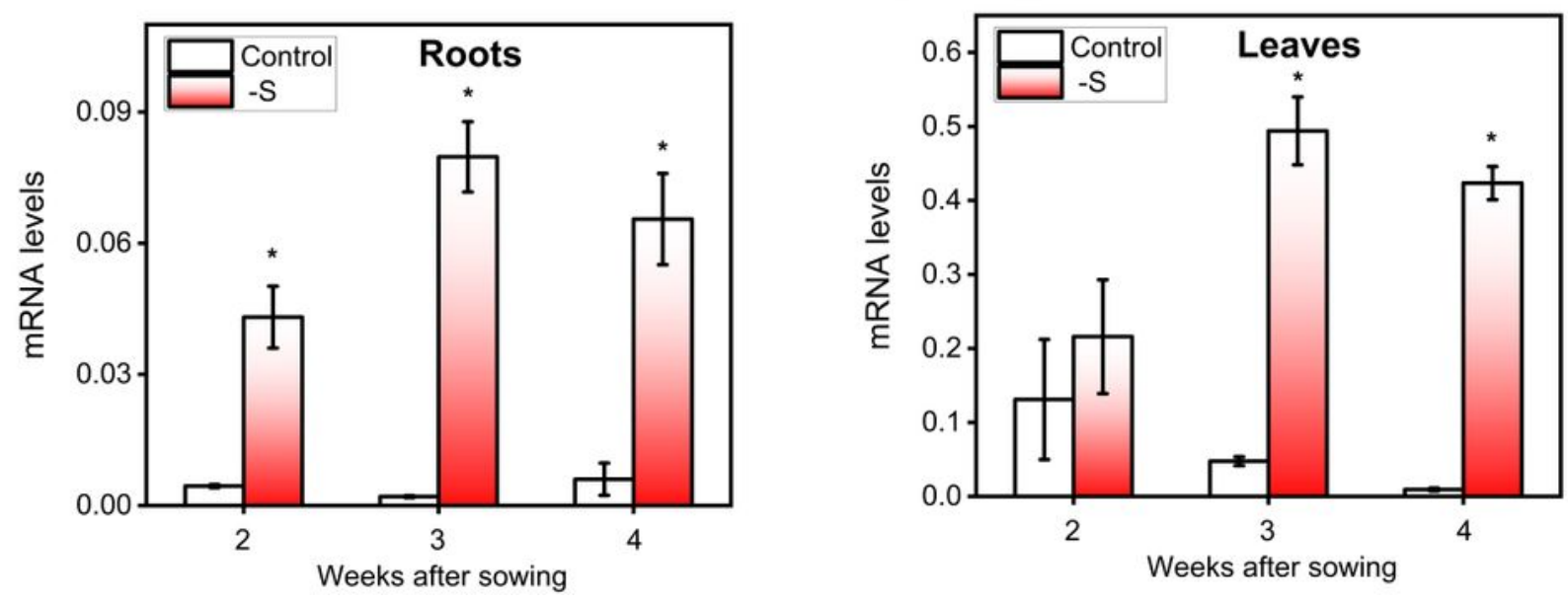

Figure 6

A gene regulatory network analysis identifies new TFs involved in the sulfate starvation response in tomato plants. A). TFs from the root regulatory network that have over-represented biological functions in their target genes. The root regulatory network was constructed considering TF-target interaction 
according to the information available in PlantRegMap and Pearson correlation threshold $>0.9$ in root samples. B) TFs of the leaf regulatory network with over-represented biological functions in their target genes. TFs and their potential targets were identified using the information available in PlantRegMap and Pearson correlation threshold $>0.9$ in leaf samples. C) RT-qPCR analysis of EIL3 TF (Solyc01g006650) in tomato plants. A Student's t-test was performed to test the significance $(P<0.05)$ of the differences between sulfate-starved and control samples $\left(^{\star}\right)$. Values plotted correspond to the means of three independent experiments \pm standard deviation. 3-5 different plants were measured for each experimental replicate.

\section{Supplementary Files}

This is a list of supplementary files associated with this preprint. Click to download.

- FigureS1.jpg

- Figures2.jpg

- FigureS3.jpg

- FigureS4.jpg

- FigureS5.jpg

- FigureS6.jpg

- FigureS7.jpg

- Figures8.jpg

- FigureS9.jpg

- FiguresS10.jpg

- TableS1.xIsx

- TableS2.xIsx

- TableS3.xIsx

- TableS4.xlsx

- TableS5.xlsx

- Tables6.xIsx

- TableS7.xlsx

- Tables8.xlsx

- TableS9.xIsx

- Tables10.xlsx

- TableS11.xIsx

- TableS12.xlsx

- TableS13.xlsx 
- Tables14.xIsx

- TableS15.xlsx 